CONTRIBUTIONS

TO THE

\title{
EXPERIMENTAL PATHOLOGY OF SPIRILLUM FEVER.
}

\author{
BX \\ H. VANDYKE CARTER, M.D., \\ BOMBAY.
}

(Communicated by Dr. James aNDREW.)

(Received December 20th, 1879-Read February 4th, 1880.)

\begin{abstract}
No. 1. The Communicability from Man to a Quadrumanous Animal.
\end{abstract}

\section{INTRODUCTION.}

THE object of inquiry was the elucidation of the pathology of spirillum fever (relapsing fever) by means of comparative experiments, which might afford fresh facilities, furnish new suggestions, or illustrate by analogy, and this object has been partly accomplished.

Attempts to reproduce the fever in pigeons, rabbits, and dogs failed here in 1877 ; in 1879 they were successful as regards one of the Quadrumana, and this animalthe Macacus radiatus of systematic authors-has been alone made use of, from its being readily procurable in 
Bombay, whither it is brought from the adjoining hills and woods. The specimens employed were all young and small (average weight $3 \mathrm{lbs}$. avoird.), fresh and in good health, and commonly males. They were kept in one large room and regularly fed; though tied apart, they were not so continuously isolated as to preclude the possibility of occasional contact.

The material employed to test the infective nature of the fever was commonly the blood, and occasionally the saliva; no antiseptic precautions were taken in collecting the blood, or during the defibrination usually practised prior to inoculation. Injections were made with the ordinary hypodermic syringe, not deeply, but into the subcutaneous connective tissue, the point of a lancet being also occasionally used; the site was near the groin, and the quantities (approximate) such as are named in the table appended. The method of inoculation did not seem to materially affect the issue.

The subjects furnishing the first and most frequently used material were native patients in the Goculdas Tejpal Hospital, Bombay; cases unselected and of ordinary character.

Attention was directed chiefly to alterations in the bodily temperature of the monkey, as ascertained by clinical thermometers placed in the axilla at two- or three-hour intervals throughout the whole day, and to changes in the blood which were to be found upon microscopic examination of specimens taken simultaneously from the digits. The temperatures were verified by myself, and both fresh and dried specimens of blood repeatedly scrutinised, whenever it appeared desirable. All notes were preserved, and the facts are stated without reserve.

The dates of experiment range from the close of February to the middle of August, 1879; their number and kind are shown in the index attached.

Dr. Henry Cook, Principal of Grant Medical College, Bombay, was good enough to verify the results of 
Experiment 10, which is a sample of the rest. The infected condition of the monkey's blood in my first essay was promptly recognised by Prof. Cohn, to whom a specimen was forwarded, and Dr. Koch has successfully repeated similar inoculations in Europe (vide the 'Deutsche Medicinische Wochenschrift,' Berlin, No. 16, dated April 19th, 1879, and No. 25, dated June 21st).

A third object, viz. to learn, if possible, the mode and place of origin of the blood spirillum, has as yet been only partially attained, but enough appears, at some inevitable sacrifice of life, to warrant the inference that this parasite grows abundantly in the spleen and liver, and probably in the vascular endothelium and white bloodcells. A large collection of parts preserved in absolute alcohol still awaits attention.

Finally, cultivation-experiments were made with specimens of the blood used in inoculation, and a note on this subject is appended. The parasitic organism may be induced to grow and expand into an open network of exceeding beauty, the spiral contour of the threads being preserved throughout. Further information on this topic may be hereafter offered to the notice of the Royal Medical and Chirurgical Society, the present details being submitted with an acknowledgment of the many imperfections inevitable to a first essay in a new and highly complicated subject.

The experiments will be described first, and then commented on. Temperature charts and specimens of infected blood accompany this paper. 


\section{PART I.-DESCRIPTION OF EXPERIMENTS.}

A. Series 1.-Positive results.

(For anpended Charts see p. 120.)

Inoculation from the Human Subject.

Invosion attack.

First Experimcnt.-N. P., a stout young man, an extra hand on duty for the previous ten days in a medical ward containing several "fever" cases, was suddenly seized with the disease, which in him exhibited its typical features of first and second attack, with an apyretic interval. On the fourth morning of the invasion, temp. $103^{\circ}$, the blood plasma was noted as being rather turbid; it contained some, but not many, spirilla, and otherwise was not abnormal in aspect. (Chart appended.)

Nos. 2 and 3.-Two healthy monkeys brought from the woods only a few hours before, and consequently quite wild, had each about fifteen minims of defibrinated blood injected into the thigh. Temperature at the time as high as $104 \cdot 4^{\circ}$, on account of their violent struggling on being handled; blood seemingly normal. Next morning the temperature had fallen to $99^{\circ}-100^{\circ}$. Twenty-six hours after inoculation one of the animals (marked W. B.) was killed to furnish specimens of the viscera at the stage of incubation. The other (marked G. P.) was allowed to live, and forty-eight hours after injection, temp. 102.2 $2^{\circ}$ a few active spirilla were seen in its blood, which otherwise appeared normal. The animal was not at all distressed, and the temperature did not rise for the next twenty-three hours, when at 8 a.m. a smart febrile paroxysm came on; at 1 p.m. the temperature was $105^{\circ} 4^{\circ}$, and at 4 p.m. $103.6^{\circ}$; in the evening at 8 it was $102.6^{\circ}$, and next morning at 8 it was $101^{\circ}$, after which there was a rise again towards night, and two days afterwards another exacerbation, also a third four days subsequently. Thence forward, for the remaining five days of detention, the temperature 
was tolerably uniform. (Chart appended.) On the morning of the day when the brief paroxysm of "fever" occurred, the blood plasma was rather clouded, contained much small protoplasm, and a very few delicate spirilla were detected on careful scrutiny; at 2 p.m., or about the acme of fever, not a single parasite was to be seen, and at 4.30 the blood plasma was clear, with very little protoplasm, no specks, and not a trace of movement. On all subsequent examinations of the blood the same negative results were arrived at, even when the temperature had risen or was rising, so that there was no evidence of a "relapse" in this case; the brief paroxysms subsequent to the specific attack were probably mere rebounds of temperature, such as are seen in other animals and in man. The monkey showed no marked signs of illness at any time; the specific pyrexia was very brief, and it will be noticed that the parasite had disappeared at its acme. The chart of W. B. is added in interrupted lines on the first and second days.

Second experiment.-H. S., an aged female mendicant, paraplegic, two months after admission into hospital was attacked with relapsing fever acquired by contagion; the invasion lasting seven days, the apyretic interval ten days, and the relapse four days. (Chart appended.) The attack was pronounced and uncomplicated. On the fourth evening of invasion the blood plasma was clear, there was seen active protoplasm, no specks, and red corpuscles normal; there were several active spirilla of the usual aspect.

2. W. T., a healthy monkey, had ten minims of this blood (defibrinated) injected into the thigh; temp. 102.2\% It was kept apart, and subjected to examination every three hours on each of the following days. The temperature was rather high, but normal in course on the second day, depressed on the third and fourth, and on the fifth there was a brief paroxysm of fever; subsequently, for fifteen days, the temperature was low and level or normal. In the night of the third day, or fifty-four hours after inocu- 
lation, the blood first showed the spirillum, which for twenty-seven hours longer was associated with a temperature rather below the mean natural, and the animal was lively. The specific pyrexia was sudden, brief, and moderate (max. temp. $104 \cdot 6^{\circ}$ ), and was not attended with much obvious distress. The blood parasite was never abundant and did not seemingly increase with the fever, being sparsely found at its acme, and disappearing within five hours afterwards; it was never again detected. The chart is appended. The sign plus-minus at 11 a.m. of fifth day indicates that in the fresh blood no spirillum was seen, and that after treatment with acetic acid some were detected. This double method was followed throughout,' but its results were particularly noticeable on this date.

Third experiment.-I-, a Mussulman sailor, shipwrecked and destitute in Bombay, who had been in contact outside with other sick men (one was in hospital at this time with spirillum fever), showed on admission all the characteristic symptoms of a first attack, which was followed by a marked relapse, and a second briefer recurrence. (Chart appended.) Two days before the first deep fall the blood-plasma was clear, fibrillation distinct and close-set; there was granular protoplasm and very many active spirilla, so that the material for infection seemed well-suited.

2. B. 3, a fresh monkey with normal blood, had five minims of the above (defibrinated) injected into the thigh ; temp. $104 \cdot 4^{\circ}$. A normal rise, slightly exaggerated, occurred next day, then a decline, and on the fourth day a very mild febrile paroxysm (max. temp. $103 \cdot 6^{\circ}$ ), which was the sole representative of the specific attack, thence forward the temperature did not vary beyond normal limits, except on the tenth day when it rose somewhat (no blood parasite visible), and the observations were discontinued on the twelfth. The blood was scrutinised regularly four times a day. Fifty-four hours after inoculation some appearances only of the spirillum were seen, which continued for eighteen hours longer, when 
during the febrile attack a single organism was detected in the fresh blood, and after treatment with acetic acid a few more; subsequently none were to be found. The chart is appended.

Remarks on the above series. In all three experiments healthy animals were inoculated with blood decidedly contaminated (once even remarkably so), and taken during the first and most pronounced attack of relapsing fever in man; yet the induced pyrexia was singularly mild, especially when least expected, namely, in the third experiment. In every instance there was a slight rise following inoculation, a depression with the advent of preliminary infection, and fever so mild and brief that it might have been overlooked. Rebounds of temperature are indicated; they are more fully shown in the longer original charts, which it did not seem necessary to reproduce throughout. The paucity of the spirillum in conjunction with very mild pyrexia is worthy of special notice.

Inoculation at First Relapse of Man.

Fourth experiment.-H. T., æt. 22, a low caste Hindoo belonging to the scavenging community (frequently affected with severe relapsing fever), showed, after a prompt crisis, a quick rebound, and two days after (eleventh day of illness) high fever set in which never left him till his death on the eighteenth day; there was lobar pneumonia on the right side three or four days at least before the end. Blood was taken by cupping on the fourteenth day (temp. $105^{\circ}$ ), and displayed a rather clouded plasma, much protoplasm, few white cells or specks, and red corpuscles mostly shrivelled, coagulation partial, and no twitching movements indicative of spirilla ; but subsequently a very few organisms were detected in a stained preparation of this blood. The man was very ill of fever, seemingly idiopathic and not specific, as the local complication had not manifested itself, and the 
blood showed no parasite until specially treated, upon this presumedly first day of relapse.

2. W. P. 2, a healthy monkey, several days in custody, had five minims of defibrinated blood as above, injected in each thigh. 'For three entire days the normal course of temperature was maintained only at lower and decreasing level, and the animal seemed to be weakened. On the fifth evening (temp. $101 \cdot 2^{\circ}$ ) spirilla were detected in the blood, and the temperature fell in the night to $99 \cdot 6^{\circ}$; it then rose next night to $102.8^{\circ}$, and on the seventh day fever was decided (temp. 104.4 ) and the parasite abounded; a sudden fall and very sharp rebound took place in the after part of the day (spirilla absent). This was the end of the specific attack, and the temperature remained low for three days; on the fourth there was a brief rise (temp. $103.4^{\circ}$, and nothing peculiar seen in the blood just after) with a quasi-normal temperature (no spirilla) for ten days longer. The acute isolated paroxysm at the close of specific pyrexia was unusual in these animals; it occurs sometimes in man. W. P. 2 showed the usual signs of illness during its short attack, and subsequent recovery was prompt; the advent of infection was here unusually deferred, even after allowing for long intervals of observation, and this may be connected with the state of his blood at inoculation from H. T.

Fifth experiment.-B. M., an adult negro of stalwart build, was admitted towards the close of invasion attack, and after an apyretic interval of five days went through a well-defined relapse ; there was no subsequent recurrence. The chart is appended. On the second evening of the relapse some blood was drawn, which presented the following characters :-Plasma clouded, fibrillation indistinct, few white cells, a little protoplasm, chiefly small, spirilla several and active.

2. R. T, a fresh, healthy monkey, had three or four minims of partially defibrinated blood injected beneath the skin of the thigh, it was then kept apart with a companion not subjected to operation. No prominent 
symptoms were noticed until five day after inoculation, when the animal appeared depressed, refused food, and was hot and shivering; temp. $105 \cdot 8^{\circ}$. The blood was then examined by gas-light; it set quickly, plasma clouded and so full of active spirilla that the whole field of view appeared in incessant motion. Next morning the fever had remitted (temp. $102 \cdot 8^{\circ}$ ), and the monkey though distressed took a little food; at noon temp. $103 \cdot 4^{\circ}$, with renewed prostration, as indicated by the lowered head and folded limbs, hiccup, rejection of all food and drink, and a sleeplessness in marked contrast with the frequent dozings of its companion; the hair was rough and staring, yet not wet. Upon examination, the blood-plasma appeared tolerably clear, fibrillation coarse and open, white cells and protoplasm scanty, a few specks ; the red corpuscles were in constant movement owing to the abundance of active spirilla. At $2 \mathrm{p} . \mathrm{m}$. the temperature had risen to $104 \cdot 8^{\circ}$, at 4 p.m. it was $103 \cdot 8^{\circ}$, and at 6 p.m. $102 \cdot 4^{\circ}$; at 9 p.m. it had fallen to $100 \cdot 2^{\circ}$, and this probably represented the crisis which thus took place some twentyseven hours after observed initiation of high fever. At 4 p.m. the blood was found to have regained its normal aspect ; plasma clear, fibrillation distinct, white cells not numerous, red corpuscles piled and quiescent; there was some granular protoplasm, and a few minute specks and filaments of indeterminate character. With cessation of fever the monkey rallied, moved languidly, and began to eat; its appetite, indeed, was ravenous; it slept. There was no rebound of temperature, and for ten days no sign of relapse ; convalescence was prompt, four ounces in weight being gained in six days; examination of blood on the second, fourth, and fifth days after fever negative. The chart in this case is withheld, being imperfect, as no night observations were made.

The companion monkey did not become infected, although in close alliance throughout; its chain caused some chafing, which doubtless induced the transient feverishness occasionally noticed; blood repeatedly examined with 
negative results. In the course of these experiments similar accidents to these little animals were not unusual.

The above was my first essay, date February 26th, 1879 , specimens of the infected blood were forwarded to Professor F. Cohn, and their character verified.

Sixth experiment.-I. S. B., a lad of 14, whose father had relapsing fever, was not admitted until the first apyretic interval, but in a specimen of dried blood taken from him at their home I found the parasite in abundance. His relapse was much shorter than his father's, and though the blood spirillum was at first very plentiful, his illness seemed to be of the mildest. On the morning of the second day (temp. $102 \cdot 6^{\circ}$ ) the liquor sanguinis was clouded and contained some large protoplasmic bodies, and there were numerous active organisms to be seen. Ten minims of the defibrinated blood were injected hypodermically in each of the following monkeys at 10 a.m. 10th July.

2. Chain, a fresh animal brought to hospital the previous night, small and wild, had normal temperature and blood. The temperature took a normal course and was rather below the mean for the first three days, it then declined and rose into a mild paroxysm prior to the onset of high fever on the fifth day; the blood was examined only twice daily, and forty-eight hours after inoculation a few active spirilla were found in the very clear plasma; next day there were more, and at last the blood was filled with the parasite. The animal was then ill, though still active, and it was killed for pathological inquiry. In this case high fever was preceded by a minor or quasi-abortive rise.

3. Rope, a similar subject and similarly treated, showed healthy blood and a temperature which, pursuing a normal course, was somewhat more pronounced than usual, until the advent of the blood parasite, forty-eight hours after inoculation. Death was then inflicted for the purpose of research at this commencement of specific infection. All charts are appended.

The concordance of date upon which the spirillum was 
first seen in both these animals, supplies evidence of uniform conditions and effects to the exclusion of accidents, such as a possible contagion; and since in the other two instances, in which more than one animal was simultaneously submitted to inoculation, a similar concordance was noted, I feel justified in relying upon the majority, at least, of my essays, which were always made in like manner.

Seventh experiment.-B. L., a destitute immigrant labourer, exhibited in hospital, after the close of the invasion, a first and second relapse with subsequent minor perturbations of temperature, all fairly characteristic of famine fever. The chart is appended. On the third day of the first relapse (temp. $103 \cdot 4^{\circ}$ ) the state of the blood was as follows :-Plasma clouded, fibrillation indistinct, white cells few, some large protoplasmic masses, no free granules, red corpuscles heaped; there were several active spirilla to be seen. At noon (temp. $104^{\circ}$ ) two drachms of blood were abstracted and defibrinated.

2. Y. T., monkey, healthy, had twenty minims of the mixed serum and corpuscles injected into the thigh; temp. $101 \cdot 2^{\circ}$. Next day the temperature rose a little as usual, and on the third day it had subsided. At 10 a.m. it was rising again $\left(102.8^{\circ}\right)$, and the animal's blood was then (forty-six hours after inoculation) found to contain a few active spirilla ; plasma rather clouded, but fibrillation seen; white cells few, some large protoplasm, no moving specks, the red corpuscles piled. The monkey looked a little drowsy, though not sick; it was now killed in order to furnish specimens of the viscera at this early stage of infection. (Chart appended.) Y. T. had previously been inoculated with dried spirillar blood, and had remained unaffected.

Eighth experiment.-F. J., æt. 28, ship labourer and immigrant, after a most pronounced first crisis in hospital, and somewhat prolonged first interval, underwent a relapse, striking from its intermittent character. On the evening of the third day, during a mild mid-paroxysm, 
the temperature was $100.4^{\circ}$ and the blood plasma was clouded, white cells very few, no moving granules, red corpuscles piled, and amongst them indications of two or three spirilla which, with the Albrecht process, were seen to be slender, though not seemingly immature. The man was not suffering much. (Chart appended.)

2. B. 1, a large monkey, rather thin and weak from the effects of a late experiment with sputum, but not now feverish, had ten minims of the above-named blood (defibrinated) injected into the thigh. Its condition as to the temperature and blood was then ascertained at threehour intervals for the next six days. The spirillum made its appearance fifty hours after inoculation (temp. 100.6 $6^{\circ}$, and was found for forty-eight hours longer, though sparingly, until high fever set in, when the parasite became abundant, and individual examples seemed to be dividing about the middle. The temperature was normal on the second day, higher than usual in the morning of the third, and with advent of the visible infection, declined considerably on the fourth and fifth days (min. $99 \cdot 4^{\circ}$ on last morning), rising at the end to $102 \cdot 4^{\circ}$, prior to the abrupt onset of pyrexia on the sixth day (temp. $105.4^{\circ}$ ), when death was inflicted for the purpose of ascertaining the state of the venous blood of different organs. The animal showed hardly any signs of illness till the last day, when it became languid and opened its mouth frequently. (Temperature chart appended.)

Ninth experiment.-S. B., father of the lad in experiment No. 6, after a slowly declining first crisis, and tolerably level first interval, had a well-marked relapse, at 2 p.m. of the third day of which (temp. 102.5 $5^{\circ}$ ) blood was drawn, and the serum allowed to separate; it contained some red discs and several spirilla, some being extended and quiescent, and others in active movement.

2. W. T. 3, a healthy monkey, had ten minims of this blood-serum injected into the thigh at 5.30 p.m., June 24 th ; temp. $102 \cdot 4^{\circ}$. The temperature declined at midnight to $101 \cdot 4^{\circ}$, and then rose steadily next day until 2 
p.m., when it reached the maximum of $106.8^{\circ}$, which was also the acme of a brief paroxysm of specific fever. It declined even more rapidly than it rose, to $100.4^{\circ}$ at midnight of this second day. A slight rebound followed, and thenceforward for twelve days a tolerably level, though low and slightly irregular, course was preserved. Here infection was very prompt, the blood spirillum being found sixteen hours after injection, and possibly being present at an earlier period, as the temperature was already $104^{\circ}$ on first employment of the microscope after inoculation; it was then abundant. Three hours after the climax of the acute isolated paroxysm (temp. 105. $7^{\circ}$ ) the parasite had quite disappeared from the blood. None but the briefest preliminary infection could have occurred in this remarkable instance, fever setting in so soon as twelve hours after inoculation. The sharpness and brevity of the attack, and its prompt decline, with absence of decided rebound, are other striking features of the case.

The experience was unusual and even unexpected, since the man, S. B., was suffering considerably, and his blood might be supposed to be highly infective. However, I should observe that blood-serum was the vehicle used (whence possibly the rapid infection of the monkey), and that in the two hours and a half after the blood was drawn and had set, some of the spirilla had ceased to be active, whence possibly the milder infection, as manifested by brief duration of pyrexia. This was the only instance known of fever coming on so soon as twelve or fourteen hours after inoculation, and it must be viewed apart from all the rest.

Tenth experiment.-R. Y., a low caste Hindoo, one of others attacked outside, was admitted into hospital just after the invasion, his chart shows only the relapse, and is appended. The symptoms were marked, but the state of the blood was at no time characteristic as usual, owing to opacity of the plasma, and sparseness of the parasite. On the fourth evening of the relapse (temp. $105 \cdot 4^{\circ}$ ) the spirilla were so obscurely indicated by a rare twitching of the red 
corpuscles, that their existence might easily have been overlooked; but on treatment with acetic acid, their presence in small numbers was fully established. The other characters of the blood were as follows :-Plasma clouded, fibrillation slow and not distinct, much protoplasm, large and small, many small rounded bodies or specks (quiescent), white cells numerous, some large granule cells, red corpuscles in small piles, and often shrivelled. The patient was affected with asthma and bronchitis.

2. W. T., a healthy monkey, had about fifteen minims of defibrinated blood injected subcutaneously in the thigh (temp. $102^{\circ}$ ); it remained well to all appearances for three and a half days, when fever was noticed at my morning visit, temp. $104 \cdot 2^{\circ}$; aspect depressed and shrunken. Blood plasma clear, very few specks, white cells not numerous, protoplasmic masses rare, red corpuscles piled and heaped; "there are vast numbers of very active spirilla, which seem to be rather larger than in man." In the afternoon at 3 the animal became worse, its head hung so low that only the radiating scalp was visible, and its general aspect resembled that of $R$. T. in the fifth experiment (No 2). At this time Dr. Cook saw the blood and became assured of the validity of the observation. At 11 p.m., temp. $103 \cdot 8^{\circ}$, still crowds of spirilla, and next morning also; no abdominal tenderness evinced, and spleen not felt; at 2 p.m. of the second day of fever the animal was killed with chloroform, in order to furnish specimens of the viscera during pyrexia; blood defibrinated after death showed abundance of active spirilla, so that the vapour of chloroform does not act as an immediate poison on these organisms. The temperature chart of this early experiment is not complete; it shows a maximum of 106.8 in the evening of the first day, a decline to 103.4 next morning, and an interrupted rise on the second day to $106^{\circ}$, when death was inflicted; the temperature was below the normal prior to fever setting in suddenly.

Eleventh experiment. - The first relapse of the man 
furnishing the material for experiment 3 was very pronounced, and on the fourth day $(5$ p.m.) the blood exhibited the following characters :-Plasma tolerably clear, fibrillation distinct, some large granular protoplasmic bodies, no specks, there were swarms of spirilla, fully formed and active, and besides some long wavy beaded filaments were seen, which possibly were immature forms.

2. W. T. 2, a monkey with normal blood, had five minims of the above (defibrinated) injected into the right thigh at 6 p.m., its temperature was $102^{\circ}$, falling in the night to $100^{\circ}$ and rising next afternoon to $102.4^{\circ}$; then sinking on the third morning to $99^{\circ}$, it rose abruptly to $105^{\circ}$, again declined, and on the fourth day of experiment ascended at about noon to $105 \cdot 2^{\circ}$, when the animal was killed. Here was a distinct paroxysm preliminary to what was probably the main attack, and the same event is occasionally seen in man. (The chart is appended.) Blood : at the first examination, fifteen hours after inoculation, a few spirilla were detected in the otherwise unchanged fluid, they appeared to be slender, but were active ; thenceforward, during the brief non-febrile period, and as well the isolated paroxysm, to the end, the parasite was invariably present, being at last very abundant. The animal did not show unequivocal signs of distress at any time previous to the last day, and even then its general state was not marked by much apparent malaise; had its life been prolonged, possibly with high fever the usual symptoms would have supervened, yet this circumstance is noteworthy (it has been seen also in children attacked with relapsing fever), as indicating that with even pronounced blood-infection the system is not necessarily overcome. Another striking feature was the rapidity of induced contamination with blood taken during the relapse, as compared with the delayed and mild results of inoculation with that taken during the invasion attack as shown in Experiment 3. I should mention that W. T. 2 had five weeks before undergone an earlier attack of induced spirillum fever (described in 
Experiment 2), and upon recovery had been returned to the bazar, whence it was again brought on the day of the present proceeding (April 26th).

\section{Inoculation at Second Relapse.}

Twelfth experiment.-R. M., a stout but scorbutic young negro of the same family as B. M., in Experiment No. 5, was admitted after the end of the first attack, and underwent a second and third in hospital. On both these occasions the blood parasite was so sparse that its presence might easily have been overlooked in ordinary examination. The second relapse consisted of an isolated paroxysm of less than one day's duration, but terminating in a fall of $10 \cdot 6^{\circ}$. At the acme of this brief attack (temp. $104.6^{\circ}$ ) the blood exhibited the following characters :Thin, devoid of fibrine, brownish in hue, but brightening on exposure, plasma clouded, little white matter, red corpuscles shrivelled and dispersed, a single spirillum after long search was detected. The patient suffered very little at the time. (His chart is appended.)

2. B. B., a healthy monkey with normal blood, had eight minims of the above (defibrinated) injected hypodermically into the right thigh at noon, 29th March; temp. $101.4^{\circ}$. For three days the blood presented no obvious change and the temperature did not vary beyond normal limits, Early on the 2nd of April, the fifth day of experiment, or under ninety-four hours after inoculation, the animal seemed well, though being kept alone it cried out occasionally ; temp. 101.8; blood plasma clear, fibrillation not distinct, very little protoplasm, and no peculiarity except the presence of several active spirilla. The temperature now declined to 99.8 at midnight, and on the next day to 98.6 at 6 a.m. ; but at 8 a.m. it began to rise and at 8 p.m. it was 105.6 ; it remained at 104.5 during the whole of the following day, and the animal was evidently ill. Early on the 5th the temperature declined to $100^{\circ}$, and 
subsequently took a quasi-normal course. The spirilla were few at first ( 1 to 4 in the field), and with the accession of fever their number increased (6 to 12), till at last they swarmed in the plasma; at 10 p.m. of the 4th April there were many of full size (temp. $104^{\circ}$ ), but at 2 a.m. of the 5 th (temp. $100^{\circ}$ ) not one was to be found. Unpromising as this experiment seemed, its results were comparable with those of No. 5, and I have no doubt that had the same early scrutiny been practised then, a similar ante-febrile blood infection would have been detected.

B. B. quickly recovered, and it was kept under notice, and daily examined for eleven days in order to ascertain the likelihood of a relapse; the result was negative. Yet on the twelfth day after fall I found that the spirillum had reappeared (temp. 102.8 $8^{\circ}$, and this was the beginning of a second attack, which had a preliminary infection period of at least thirty-six hours, and culminated in a smart febrile paroxysm (temp. $105.2^{\circ}$ ), of twenty-seven hours' duration, the parasite, at first sparing, was then abundant ; there was hardly any constitutional disturbance, and return to the normal state was prompt, and maintained for the next thirteen days. I am unable to assign the cause or character of this fresh attack, seemingly it was spontaneous, and the probability of contagion remote, yet with other contrary experience I cannot view the event as a true relapse, unless it be that repetitions of fever by auto-inoculation are, in the monkey, subject to irregularities not perceptible in the human subject. Contagion was not proved amongst these animals when it might have been looked for, but it had possibly occurred in this case. (Two charts are appended.)

Inoculation at Stage of Incubation.

Thirteenth experiment.-S. B. (vide Experiment 9) had a somewhat prolonged first interval, and on the ninth day, or two days before the relapse (temp. $98^{\circ}$, and health 
fair), his blood was described as clear, white cells few, protoplasmic bodies small, red discs normal ; after treatment with acetic acid only a few specks and short rods were noticeable as possibly unusual ingredients.

2. R. T. 2, monkey, had ten minims of the above (defibrinated) hypodermically injected into the thigh; it remained unaffected to all appearance for fully ten days, the temperature being level, except on the seventh day, when a brief rise to $103 \cdot 4^{\circ}$ took place; the blood was unchanged. After so long an interval this experiment was regarded as a failure, and another injection was about to be made, when I faund in the blood (temp. 102.6) several active spirilla, and the animal appeared sluggish, though not ill. About twenty hours afterwards a brief febrile paroxysm occurred, which lasted six hours and ended abruptly. The parasite was detected prior to and at the acme (temp. $1054^{\circ}$ ), and had disappeared three hours afterwards. The monkey was ailing for the time, but soon and permanently rallied.

Respecting this irregular example, I have to observe that four other monkeys were inoculated on successive days of the same apyretic interval. $R$. T. being the third in this series, and in all the rest the result was negative; further, it would be quite extraordinary if so long a period as ten days' incubation were conceivable in these animals, and $I$ am therefore inclined to suppose that in the present instance R. T. 2 had acquired the fever it displayed by means of contagion from W. T. 2., (vide 11th Experiment), although I had given orders for the latter to be kept apart; were this so the incubation period would be reduced to five days, and the anomaly would be accounted for. Observation has led me to infer that, like men, these quadrumana differ individually in their susceptibility to infection, and though direct attempts were made to test contagion amongst them ineffectually, I think it probably took place in the present instance, and in the 12th and 17th Experiments as the vera causa of the second attacks then noticed. This opinion is inferential only, and in the 
appended table I have felt bound to give the figures actually found.

InOCULATION FROM THE MONKEY.

Stage of High Fever.

Fourteenth experiment.-W. T. (vide Experiment 10). On the first day of observed pyrexia (temp. $104 \cdot 2^{\circ}$ at 11 a.m.), and general symptoms of illness manifest, the state of the blood was as follows:-Plasma clear, fibrillation slow, white cells and protoplasm scanty, a very few moving specks, and red corpuscles piled and heaped; there are vast numbers of most active spirilla, which appear to be rather larger than those of human blood.

2. R. T., monkey, in fair condition, though rather thin in consequence of an earlier attack (vide Experiment No. 5), this day, March 16th, being the twelfth after the fall, and no sign of relapse being manifested during the interval, a minute quantity of blood was taken by puncture from the thigh of W. T., as above, and inoculated with the same lancet in the thigh of $R$. T., the clot, not larger than a pin's head, being introduced beneath the skin; no bleeding ensued. The animal remained well, and its temperature was tolerably uniform until midday of March 20th, or four complete days after inoculation, when an abrupt rise took place; the attack thus initiated lasted sixty-six hours, and was continuous; the maximum temperature of $1064^{\circ}$ was noted about midway, the decline occurred at 6 a.m. of the 23rd, with a prompt descent to $100 \cdot 6^{\circ}$. The general symptoms of the fever were the same as seen on other occasions, and the monkey was killed at the fall in pursuit of further inquiry. (Chart appended.)

This early example of communicated fever was unmistakably clear, and it is noteworthy as proving that no protection against reinfection is afforded by even a recently foregoing attack; it also shows that no predisposition to a recurrence, as estimated by comparative vol. LXIII. 
length of incubation period, can be said to result from such previous attack; and, lastly, it demonstrates that a very small quantity of blood, or hardly more than the amount of lymph commonly employed in vaccination, will suffice, when introduced beneath the skin, to convey a pronounced spirillar infection.

Fifteenth experiment.-W. T. (vide Experiment 10), on the second day of observed fever, or upwards of thirtytwo hours after its initiation, the defibrinated blood, obtained after death by chloroform, was found to contain abundance of active spirilla, which were seemingly unaffected by the inhaled narcotic, and it was therefore employed for further testing the communicability of spirillum fever at first remove from man.

Three monkeys had each a few minims of blood minus fibrin subcutaneously injected in the same manner and hour ; of these two were fresh acquisitions from the bazar, and the third (C. below) was the companion of R. T. (vide Experiment 5), which, although in closest propinquity to that fever-stricken animal, had never shown any sign of contagion. The results are briefly described below, and the fever charts are appended entire, with the exception of the first two days, when night observations were not complete.

2. N. W. T. (also termed " biter" from its viciousness). The temperature rose beyond the mean for a few hours, and then became nearly normal; on the third day it rose again simultaneously with that of the two other animals, and rather higher, being at 11 p.m. $106^{\circ}$, and the monkey still very wild; the blood contained many spirilla. Next day the temperature subsided a little, being $103.8^{\circ}$ at 11 a.m.; the animal now seemed ill; its blood was highly contaminated, and death was inflicted when there was every prospect of the attack continuing as in its neighbours.

3. R., the other fresh monkey, showed but little variation of temperature for forty-six hours, when fever came on rather gradually but decidedly $\left(104 \cdot 2^{\mathrm{C}}\right.$ in the first 
evening, and $106 \cdot 2^{\circ}$ on the next) ; then, after a morning abatement, again rising (third day), and so again (fourth day), when the crisis took place (fifth morning) in a decline to $1.01 \cdot 4^{\circ}$ at noon. Apparently this ended the specific febrile attack, which had thus lasted for about ninety-four hours or four complete days; and now began a sharp and prolonged rebound, the temperature at 4 p.m. having suddenly risen to $107 \cdot 2^{\circ}$, and this exacerbation persisted throughout the next day (at 4 p.m. temp. $106 \cdot 2^{\circ}$ ); but on the third the body heat declined at first slowly, then rapidly, until the animal died at 4 p.m. The blood was repeatedly examined throughout the entire illness, and found to contain numbers of the parasite on the first day and second, and even at the considerable decline on the morning of the fourth day (temp. $102 \cdot 2^{\circ}$ ), when it swarmed with clusters of spirilla. On the true fall occuring next day at noon, not a trace of the growths was to be seen even with the $\frac{1}{24}$ in. immersion lens, or indeed any abnormal appearance. The secondary pyrexia, or rebound, was also marked throughout by an absence of the spirillum, and so its real character was made manifest. At the beginning of its illness the monkey was very quiet, and sweating of the palms was noted; next day, though fever was high, it sat up and took food in the morning, but at noon it joined the other two, and all huddled together, being evidently very sick. On the third day both survivors presented the characteristic aspect, drooping, pallid, and shrunken, refusing rice, nibbling only at fruit, and drinking water, though not with eagerness. At the fall the symptoms were mitigated, but with the renewed pyrexia, shiverings and depression were noted, and finally prostration, semi-consciousness, rapid breathing, and lowering of temperature till death. An autopsy was made and the parts preserved. I may here observe there was found no localised inflammation sufficient to account for the fatal termination, unless some extreme redness of the small intestine be regarded as inflammatory; the 
animal seemed to die of fever as human subjects occasionally do.

4. C., a monkey, some time under observation, and affected with an accidental sore, but never the subject of experiment, showed a high temperature shortly after inoculation, which had subsided next day. On the third the temperature again rose, but somewhat more gradually than in the other two animals, declining in the morning, to ascend permanently at night to $106 \cdot 2^{\circ}$. On the fifth day there was a morning decline and evening rise, and so on the sixth, when the acme of $106.8^{\circ}$ was attained a few hours before the critical fall to $101.6^{\circ}$ in the morning of the seventh or following day. Except at the beginning, the course of the pyrexia was similar to that in No. 3. The animal was lively on the second morning when the blood was found to be full of spirilla; soon after it became sick, and remained so till the fall. On the last day, at 5 p.m., the blood contained many active parasites; an hour later there were none, and at 8 p.m. the fever reached its turning point. I had intended to watch the case by minutes, but was called away to perform an amputation; sufficient was seen, however, to show how abruptly the blood parasite disappears at this period of febrile crisis, and the observation was even frequently confirmed in the wards of the hospital. A prompt and pronounced reaction of temperature followed the cessation of specific pyrexia, and it was prolonged for four days, slowly subsiding at the last, and the monkey then quickly became convalescent. The blood was free from visible contamination at this time, and no relapse followed during the eighteen days after crisis; the animal was closely watched.

The above experiment is decisive of the inoculability of spirillum fever, of the similarity of symptoms under similar conditions, of the greater severity of the fever induced by infection at inrst remove, i.e. from monkey to monkey after a prior infection from man; and, finally, of the similarity of the comparative phenomena to the human, with the 
exception of the relapse, so-called, which was not seen in the lower animal surviving.

Sixteenth experiment.-From monkey No. 2 of the last experiment, the blood taken twenty-six hours after initiation of pyrexia was swarming with spirilla ; it was allowed to stand for three hours and a half, and then had separated with considerable distinctness into clot and serum. The serum was found to contain many moving parasitic organisms and a few red discs unchanged, with no other visible particles; the clot was dark and firm.

2. B. T., No. 1 in the chart, had ten minims of the slightly-tinged serum injected beneath the skin of the thigh at 5.30 p.m. of 20 th March; temp. $103^{\circ}$. The body heat rose the next day to somewhat above the normal mean, and still more on the third and fourth days, descending to the mean at $8 \mathrm{a.m}$. of the fifth day; it then rose promptly to $105.4^{\circ}$, and continued high on the following day; early on the seventh the critical fall took place, and very soon after a sudden and sharp rebound (temp. $107^{\circ}$ ); on the eighth day the temperature declined, at first moderately, and at last very quickly, the animal dying this night (the last entry, temp. 96.4\%). The blood was examined at intervals, and the spirilla were found before fever set in, being then few and small; with rise of temperature they became numerous, the animal not seeming to suffer much. On the sixth day, with continuous fever, the parasites were fewer, seeming gradually to decline at first, then towards the end of the specific attack they were abundant and aggregated in clusters; at the fall on the seventh day all traces of them had disappeared; with the rebound of temperature the blood was found to be loaded with plasmic material only. The animal was very ill, as if overpowered by depression, the ears and extremities being cold, and the head and trunk very hot; no spots could be seen on the skin; it remained in this state, the body cooling, until its death. The stomach and small intestines were apparently much inflamed, and there were some hæmorrhagic spots. 
3. O. T. Two bits of the above blood clot, not larger than a pin's head, were introduced beneath the skin of the left thigh; they had been slightly washed in a weak solution of sulphate of soda, and were not examined microscopically, it being supposed that a few spirilla would be still entangled in the fibrin meshes. The temperature maintained a quasi-normal course for two days, and on the morning of the fourth a few active spirilla were detected in the blood. Pronounced fever did not come on till the following day (temp. $105 \cdot 6^{\circ}$ ), it was less next day, and on the seventh, when an exacerbation took place (spirillum present), which ended in the fall. After a few hours a sharp rebound set in, and at 4 p.m. of the eighth day the temperature was $106^{\circ} 8^{\circ}$. This renewed fever was a veritable relapse, resembling in form and duration, and exceeding in intensity, the preceding attack, but distinguished from it by the entire absence of spirillar bloodinfection; at the close the animal was so exhausted that it died. At the autopsy I found some inflammation of the stomach and small intestines; there were some petechiæ on the heart.

Remarks.-The similarity of results in this experiment is manifest, even in particulars, and the series may also be compared with the last, their common feature being increased severity of infection with maintenance of all characteristics. The rebound, or secondary fever, or "relapse," properly so-called, was present in all these instances of inoculation at removes from man, and I infer that the infective property of the blood was increased at each successive remove. As both these last animals died, there seemed no occasion to pursue this subject by means of fractional inoculation, and my experience had not been such as to warrant confidence being placed in dates and changes of temperature as criteria of intensity of blood poisoning in this particular form. I note that although the infection in Experiment 16 was sufficiently intense to lead to death, yet the incubation-period of the fever was not shorter than the mean, nor was the specific tempera- 
ture unusually high ; and Experiment 20, and those made with saliva, show that other organic poisons operate differently to the spirillar. Here the varying state of the blood at different stages of the "fever," and probably idiosyncrasy of subject, are elements of calculation as yet not enough known. There could hardly be a more intense degree of blood-infection than was displayed in my experiments, and all the deaths occurred during secondary fever; perhaps some elucidation of the character of this last-named sequela might be elicited by the fractional method.

Seventeenth experiment.-W. B., in Experiment 1 (vide chart), had twenty-six hours previously to furnishing the infecting material used below, been injected with spirillar blood from a human subject, which was proved (in its comrade, G. P.) to be capable of conveying disease. In this material no sign of the parasite was detected; plasma rather clouded, fibrillation visible, white cells few, little protoplasm, specks few and quiescent, red discs piled and heaped.

2. E., a healthy animal with normal blood, had a few minims of the above defibrinated blood injected in the usual manner at noon, March 25th; it remained well till the 29th, the temperature being tolerably uniform in its daily oscillations, though rather higher than usual at maximum $\left(103^{\circ}\right)$. The blood was examined twice daily (excepting on the 28th), and at 10 a.m. of the 29th, or ninety-two hours after injection, it was found to contain a few active spirilla without being otherwise peculiar. Next day there occurred a smart febrile paroxysm (temp. $106 \cdot 2^{\circ}$ ) of about eight hours' duration, and soon after its acme the blood was found to be free from the parasite, only some moving granules being detected; the temperature then declined to $101^{\circ}$. There seemed no reason to doubt that this brief attack was due to artificial infection, and hence the inference that the germs of specific pyrexia are not necessarily represented by the blood parasite.

Relapse.-E. continued well for six days, or about 150 
hours, during which time the temperature oscillated between $100^{\circ}$ and $103^{\circ}$, and whenever examined (once or twice daily) the blood was normal in aspect. In the afternoon of 6th April, one or two active spirilla were detected (temp. $103^{\circ}$ ), and again next day (temp. 102.2 ${ }^{\circ}$ ). On the 8th, or thirty-six hours after their first advent, a smart febrile paroxysm took place (temp. 105 ) which lasted ten or twelve hours; at its onset the parasites became numerous, and at its decline they had all disappeared. The animal looked pale and thin at this time; it was kept under observation for eleven days longer with negative results. (Charts appended.)

Remarks.-The interval between the first and second attacks of fever was longer than the first incubating period as nine to five, and this points to a character of relapsing fever in man; both attacks were alike in the preliminary non-febrile infection, and in the form and duration (nearly) of pyrexia, and this circumstance also seems in favour of their essential connection; the alternative view is that contagion was the cause of the second attack. Compare with Experiment 12, where the "relapse" came on nearly twice as late, thus showing a suspicious want of conformity which is of the rarest in man.

As regards the invasion-attack, this experiment corresponds to No. 13, in which also incubation-blood seemed to prove infective; both attacks were mild, and so far alike, yet there is a wide discrepancy in their dates of onset, which it is difficult to comprehend, except upon the supposition that the spirillum fever of the monkey is liable to hidden modifications hardly consistent (as it might appear) with the open manifestations in the human subject; here, too, the alternative view was that of incidental contagion. 
Series 2. Experiments furnishing negative results.

The fact of communicability under certain conditions being established, I proceed to narrate some other experiments made in similar manner, which may assist in elucidating the nature of these conditions.

Failure of Spirillar blood to infect.

1. Of man, at invasion attack (a).

Eighteenth experiment.-K. G., a pauper immigrant, æt. 55, had a severe attack of spirillum fever, which was said to be the first and seemed to be much prolonged (fourteen to fifteen days) ; the end only was seen in hospital, and it was accomplished by slow descent extending over three days, during two of which the blood parasite was abundant. Immediately afterwards a sharp rebound took place, with head symptoms, and the patient died (cerebral hæmorrhage). His daughter was in hospital, and also died after her attack (thrombus in the femoral veins); the wife had had "fever" a short time before, and had recovered. There were no other members of this family. On the first day of decided decline (temp. 100.4 ${ }^{\circ}$ ), the blood contained many spirilla, and a minute freshly clotted portion was used for inoculation. (Chart appended).

2. Y. T. 2, a monkey, with normal-looking blood, had the above fragment introduced beneath the skin of the thigh (temp. $102^{\circ}$ ). The same evening the temperature rose to $102 \cdot 6^{\circ}$, when the blood was still normal (red discs somewhat irregular); four hours later the temperature rose to $105^{\circ}$, falling promptly to $101 \cdot 8^{\circ}$ next morning (blood healthy), and subsequently for eight days there was only such oscillation of body heat as might be referred to the presence of sores on the body, the blood remaining unchanged in aspect. 
Hereinoculation failed, though made after the same fashion as in the successful Experiment 16, No. 3 ; there is no reason to suppose that the brief rise after inoculation was of specific character, and upon consideration, I infer that when the spirilla, as a rare event, persist till near the end of the crisis, their presence does not necessarily imply infective quality of the blood. Under artificial cultivation, I found that at this time the parasites in the blood of K. G. (who was unusually ill) did not grow as other specimens had done, whence it is probable that these organisms do not themselves evolve the germs of future broods, except under certain conditions which do not pertain to the close of a febrile attack. Yet upon the hypothesis that early " broods" or "crops " of the parasite usually leave behind them, in the blood itself, the germs of a succeeding growth to be developed with the "relapse," it might be supposed that blood at or near the "fall" would be both infective and culturable; the contrary being the case, I infer that the "germs" are lodged (if not produced) outside the blood-current. Perhaps the conditions vary, for reproduction is not invariable.

\section{At first Relapse (b).}

Nineteenth experiment.-Blood taken on the third day, or the day after the successful attempt described under Experiment 5, from the man B. M., had the following characters:-Plasma not clear, a few white cells, some moving granular protoplasm, many specks, and several spirilla in active movement.

W. and B., two monkeys in good health, had the entire blood injected, namely, a few minims beneath the skin of one, and the same into or around the femoral vein of the other; no local or general ill effects followed, both animals continuing unaffected for eight days. Special notice was taken on the fifth day, or the date of fever in the earlier experiment, and I conclude that the present attempt 
was a failure. Why it should have been so is not manifest, for both animals were afterwards successfully inoculated, and the material employed did not appear to differ materially from that used the day before with signal results. I notice, however, that at the time the temperature of the man B. M. was at its highest $\left(105.8^{\circ}\right)$-and it is probable that at high temperatures, whether mid or final, the parasite loses something of its dynamical activity-the man was sweating when the blood was abstracted; and upon examination of the latter by the acetic acid process, I did not find so many parasitic organisms as was anticipated. The alternative view would be that some fault or accident in either procedure or instrument had intervened to prevent infection; none was perceived.

Twentieth experiment. - The patient named in the successful experiment, No. 4, had, two days at least before his death, pronounced pneumonia (it seemed to be of pyæmic character from the post-mortem examination) in conjunction with spirillum fever; thirty-six hours before decease (temp. $104 \cdot 6^{\circ}$ ), nothing definite of peculiar aspect was seen in the blood as ordinarily scrutinised, but I found upon treatment with acetic acid a very few spirilla, besides many small protoplasmic particles-infection, therefore, was possible.

2. R. T., a fresh, healthy monkey, had ten minims of the above, defibrinated, injected into the left thigh. The effect was high and continuous fever ending in death on the fifth day, and the blood never showed any signs of specific contamination; twelve hours after inoculation, the temperature rose and shortly attained $105.8^{\circ}$, it remitted on the third and fourth morning, and the animal died on the fifth, there being some local sero-purulent infiltration above the side of injection, and possibly septicæmia. Here, I suppose, there existed a poison in the patient's blood, which in the monkey over-powered the spirillar infection, checking its development and inducing a fatal toxæmia. A similar instance afterwards occurred (vide No. 24), and I do not regard either merely as exceptions 
to the rule of spirillar reproduction; both were complicated cases and otherwise interesting as showing the antagonism of blood poisons.

Twenty-first and twenty-second experiments.-S. B. (vide No. 9) displayed the relapse ten days after complete fall; as usual, it began abruptly, and blood was taken morning and evening of the day of onset.

1. The morning temperature was $97 \cdot 4^{\circ}$, pulse 84 , the patient convalescent, the fresh blood was noted as containing clusters of white cells, very little protoplasm, but numerous active granules, and by the acetic acid process a few small yet distinct spirilla were also detected.

2. G. T., a healthy and excitable monkey, with normal blood, had ten minims of the above injected after defibrination ; for five or six days afterwards the temperature showed marked daily paroxysms with occasional high range (as $104 \cdot 4^{\circ}$ on the sixth afternoon), and yet the blood, examined twice daily, furnished no evidence of spirillar infection. The animal itself continued well. Observations were made until the fifteenth day, with like negative results, and it must be supposed that the parasitic organisms were too few or too immature to induce specific fever, supposing that they represent the infecting agent, the daily perturbations of temperature may be attributed to the excitability of the monkey.

3. The man's relapse set in at 4.30 p.m., and at 5.30 the temperature was $102^{\circ}$, pulse 100 , the blood then showed a few spirilla, with small protoplasm and some granules ; ten minims, defibrinated, were injected.

4. F., a fairly healthy monkey, but so restless that the chain it was bound with, caused, by chafing, a large ulcer round the waist, and feverishness in consequence. The chart shows this mild pyrexia to be continuous, and as the blood was carefully examined daily with negative results (only protoplasm and white cells abounding) I conclude that there was an entire absence of specific infection in this experiment also. Possibly the local irritation may have rendered the animal's system unfit, or the injected 
parasites were too few or immature; two days later the blood of this patient proved to be promptly infective in another monkey (Vide No. 9).

2. Failure with Spirillar Blood of the Monkey.

\section{During Fever.}

The animal, No. 2 in Experiment 6, furnished bloodserum containing many active spirilla at the time of its decease.

Twenty-third experiment.-Four minims were injected beneath the skin of Y.T. 2, a monkey previously the sub. ject of ineffectual trial and troubled with sores. The usual observations were made for eight days continuously, and I became assured that no infection took place. Soon after, Y. T. 2 lost health and died with nervous symptoms. It was probably an unhealthy animal, and seemed to be insusceptible to this particular fever.

\section{During Preliminary Infection.}

Twenty-fourth experiment.-Blood taken from B. B. twenty-four hours after the relapse was noted, temp. $102^{\circ}$, and several small but active spirilla being present (vide Experiment 12), was introduced on the point of a lancet beneath the skin of B. 1. By oversight, next day B. 1 was injected with human saliva from a fever patient (first interval), and immediately high pyrexia set in which was not of the spirillar character. This experiment, therefore, was a complicated one, and it shows only that one kind of blood contamination is capable of overcoming or preventing another. (Vide also No. 20.)

Twenty-fifth experiment.-From R. T. 2 (vide No. 13), whilst the temperature, though rising, was still not in excess of the normal, a minute quantity of blood containing several active spirilla was taken, and on the point of a lancet introduced beneath the skin of the thigh of B. B. 
This animal had been previously the subject of experiment, but was now in very good health. No result of marked or specific character followed during the next eight days, and why this was so did not appear; perhaps there was some defect in the manipulation, or the blood of R. T. may have been in a peculiar condition, for brief and high fever came on in six hours, and then the spirilla disappeared.

\section{During the Earlier Incubation Period.}

Twenty-sixth experiment. - Twenty-four hours after inoculation of the animal mentioned in No. 3, and whilst the temperature and blood were seemingly normal, two half-drops of blood were taken, and on the point of a lancet introduced beneath the skin of both thighs of B. 2, a healthy monkey, though troubled with sores on the body which made it fretful. The temperature rose on the next and succeeding two days, but was within normal limits for the following six; and the blood regularly examined never showed any sign of spirillar infection. Here inoculation with blood at early incubation stage failed to act, whilst in Experiment 17 it seemed to be efficient.

This essay forms a transition to another negative series of attempts I next proceed briefly to narrate.

B. Series 3.-Experiments with Non-spirillar Blood, with Dried Spirillar Blood, and with Saliva.

To test further the validity of the instances of successful inoculation, I employed, in the common method, and upon similar subjects, the blood taken at the "fall" or crisis of the spirillar attack, also at dates preceding the expected "relapse," and, finally, during the secondary fever or " rebound" which sometimes follows specific pyrexia.

Seeing that a spirillar organism similar to that detected in the blood is to be found in the saliva or fluids of the mouth, I also made use of this sputum as material for 
injection, taken from both febrile and non-febrile subjects.

Lastly, blood that had been dessicated was employed in other experiments, with a view of ascertaining whether or not the blood containing spirilla or their germs (supposed) was infective after being dried up.

\section{Blood at "Fall."-Man.}

Twenty-seventh experiment.-S. B. (vide No. 9), at the close of first attack, presented characteristic symptoms with a critical fall so prolonged as to resemble decline by "lysis." Near the end the morning blood (temp. 101.6 contained many active spirilla, whilst that of the evening (temp. 98.8 ${ }^{\circ}$ was absolutely free from the parasite, and served for the following operation.

2. R. T. 3, a new and healthy monkey with normal blood, had ten minims of the above, defibrinated, hypodermically injected in the thigh ; next day the temperature rose to $104 \cdot 2^{\circ}$, and declined to $99 \cdot 8^{\circ}$ on the following morning; afterwards it was tolerably level, subsiding slightly for the nine days of observation; the animal appeared to be unaffected. The blood was carefully examined, and the result was negative. The rise of temperature on the second day was attributable to irritability of the subject, which for a few hours once escaped custody altogether.

\section{Monkey.}

Twenty-eighth experiment.-R. T. had a well-defined attack of spirillum fever (vide No. 14) terminating with a prompt fall (temp. $100 \cdot 6^{\circ}$ ), three hours after which it was killed and furnished blood at this time with clouded plasma, partial fibrillation, few white cells, little protoplasm, large red discs normal and no movements.

2. Chain 2, a fresh restive animal, had twenty minims of the above, defibrinated, injected into the thigh (temp. $102 \cdot 6^{\circ}$ ), blood normal. The temperature rose in the night 
but speedily subsided, and thence maintained a uniform course till the seventh day, when there occurred a brief febrile paroxysm ; afterwards it was level till the sixteenth day. The blood was repeatedly examined with negative results; the exacerbation noted seemed clearly attributable to irritation of the chain, ending in an ulcer, and it was neither attended nor preceded by specific changes in the blood.

So far as they go, these two experiments are decisive as regards the non-infecting properties of the blood taken at or immediately after the crisis of an attack of spirillum fever.

\section{Blood at "Rebound."-Monkey.}

Twenty-ninth experiment.-B. T. 2, of trial No. 16, after a well-defined attack of specific fever, and at the fall a pause of three hours, had a sharp rebound of temperature from $102^{\circ}$ to $106 \cdot 6^{\circ}$; the animal was then very ill, and eventually it died. The state of the blood at the onset of this secondary fever was as follows :-Plasma clouded, fibrillation partial, much protoplasm, some large granule cells, several white cells, red discs heaped, shrunk, and blended, no spirilla.

2. W. T., a healthy animal with normal blood was inoculated in both thighs with the dark, thin blood of B. T. 2, as above; temp. $102 \cdot 4^{\circ}$, rising towards the close of the second day but subsiding on the third and fourth, and again rising on the fifth and sixth. The blood was repeatedly examined and always with negative results, and the experiment shows the non-infecting character of blood taken after the specific fever, when even more pronounced secondary fever occurs. I consider, also, that the nature of this rebound is indicated by the present trial, in so far as its relationship to septicæmia or other special blood contamination is concerned, for the effect of injection was here nil. W. T. was subsequently inoculated with success (vide No. 2). 
Blood during the Apyretic interval, prior to expected Relapse.-Man.

Thirtieth and thirty-first experiments.-A., a man, admitted with high fever (specific), which, according to his account, was a first attack, displayed the usual level apyretic stage following the crisis, and on the third day his temperature was $98.4^{\circ}$, and his blood free from any signs of the parasite.

Ten minims after defibrination were injected into the thigh of a monkey (Chain 2, of No. 28), temp. $102^{\circ}$; next day there was a rise, on the third none, and on the fourth a smart paroxysm (temp. 104.8 ${ }^{\circ}$ ); afterwards the temperature continued to be level for ten days. The blood was frequently examined and nothing abnormal was detected in it, excepting on the morning of the fourth day, just before the paroxysm above alluded to, when small curved filaments were seen, which at the time I regarded as resembling imperfect spirilla ; from subsequent experience, I should not lay any stress upon such appearances, unless in the same specimen undoubted parasitic organisms were also to be found.

On the sixth morning after the fall, the temperature of A. was $97 \cdot 4^{\circ}$, and blood only rather clouded.

A second fresh monkey (B. 1, of No. 24), with normal blood and temperature $101 \cdot 4^{\circ}$, was injected similarly to the first; slight exacerbations of temperature followed on the first, second, and fourth days, and on the eighth the observations were closed, all having been negative in results, including the frequent scrutinies of the blood.

The man himself had no relapse, nor did his blood display any marked changes at the dates when the second attack usually supervenes.

Thirty-second, thirty-third, and thirty-fourth experiments. -The patient named in No. 9 had quite rallied after the invasion attack, and showed a level (temp. $97^{\circ}$ to $98^{\circ}$ ), and somewhat prolonged first apyretic interval. In anticipation of the relapse, I abstracted and used for vOL. LXIII. 
inoculation blood drawn on the seventh, eighth, ninth, and tenth days after the main fall and disappearance of the parasite. The same process was followed on each occasion, the morning blood being taken, promptly defibrinated, and about ten minims injected subcutaneously into the thigh of a healthy monkey. The four animals were then kept apart, the temperature every three hours taken and recorded, and their blood scrutinised twice daily for ten days.

The result was in every instance negative, the temperature charts showing nothing peculiar and the blood being unchanged. That there was no want of susceptibility in three of the animals is apparent from two of them being afterwards successfully inoculated, and the third is that case described in Experiment 13, where contagion probably occurred ; the fourth monkey, however, seemed to be impervious to infection (vide Y. T. 2., in No. 23).

This series is continuous with that narrated under Nos. 21 and 22, which was concerned with the onset of the relapse, and furnished similarly negative results, whilst No. 9 shows successful inoculation with blood taken during the recurrent attack. I consider Experiments 30, 31, 32,33 , and 34 , prove that the non-spirillar blood of the apyretic interval in man is incapable of conveying infection.

\section{c. Series 4.-Experiments with Dried Blood.}

At the late period in the epidemic at which my inquiries began, the supply of infecting material from hospital patients was only intermittent, and as the source from animals was necessarily of brief duration, I had early to consider if spirillar blood might not display its characteristic property in the dried as well as in the fresh state. Several specimens of blood proved by experiment to be infective, were accordingly dessicated with care in thin layers, either simply under cover at temperature of the air (about $80^{\circ} \mathrm{F}$.), or over sulphuric acid, or over caustic lime, and some of these attempts at preservation were so far successful that the dried blood was found by the 
microscope to show, when moistened with distilled water, the parasitic organisms hardly altered in aspect, and but little intermixed with new growths. This material suspended in water was freely used, and I also added in two instances some acetic acid with a view of better liberating the spirilla or their germs from the albuminous substance in which they were embedded. These essays to conserve the blood intact without the aid of antiseptics (the use of which seemed undesirable) have all failed; they form the Series No. 35 to 42 inclusive.

Thirty-fifth experiment.-Blood taken at the crisis of a well-marked first attack in a man, dried in vacuo, and hermetically sealed was, after an interval of eight months, inoculated in B. T. monkey, about a grain's weight being used ; the result was negative.

Thirty-sixth experiment.-Blood taken on third day of first relapse, dried under cover, and kept for five days only, was inoculated in C. monkey. The temperature rose for the first three days, and then became normal; there was no visible change in the blood of the animal. The same material produced no effect in two men who had small quantities placed under the skin. In both the above instances the monkeys readily acquired the fever upon inoculation with fresh blood.

Thirty-seventh experiment.-The blood of W. T. monkey, known to be highly infective, was dried over sulphuric acid, and two months afterwards employed on B. B. When moistened with water some bacteria were found, but the spirilla were numerous, and on the addition of acetic acid appeared to be unchanged. The softened fragment and drop of fluid were introduced beneath the skin of the thigh, the temperature was taken, and blood examined with care for twelve days in succession, and there was no sign of illness or spirillar contamination.

Thirty-eighth and thirty-ninth experiments.-The blood of man (first relapse) and of a monkey, known by experiment to be infective, was dried without undergoing any apparent change, and nearly two months afterwards 
inoculated in two monkeys, which never showed any evidence of being affected thereby. In the man's blood no trace of the spirillum was detected after desiccation, but that of the monkey showed them distinctly. Bacteria were present in a dried condition, and these produced no effect on inoculation.

Fortieth and forty-first experiments.-The same materials were moistened with acetic acid, and so rendered soft and translucent; they were then used for inoculation in two fresh monkeys, and still without result.

These failures contrast with the success so readily obtained with the preserved blood of animals affected with the so-called splenic fever; and they serve to show that the Spirochæte possesses more delicate physiological properties than the Bacillus.

\section{Series 5.-Inoculations with Saliva.}

The following experiments display a notable contrast with the results of the previous successful inoculations :

Forty-second experiment.-About the same time as the blood was employed of the patient No. 1, I made use of his saliva for injection. High fever being present the fluids of the mouth were scanty, the saliva had a milky aspect and gave a whitish sediment; it contained large epithelial scales, much granular matter, and many active bacterioid forms, including the spirillum. As contrasted with the parasite in the blood, this of the saliva was larger or thicker and more sluggish, never twisting or lashing into knots and rings, and never clustering together; perhaps the more tenacious medium was concerned here. I observe that the Spirochæte proper of Ehrenberg, as I found it in tank water, is much larger than either of the above, and always preserves its spiral contour in movements, however active; whereas the organisms in man straighten out in their contortions and display the screw-like form only when nearly or quite quiescent.

About twenty minims of the above fluid were hypo- 
dermically injected in the thighs of two fresh and healthy monkeys.

2. B. (temp. $102 \cdot 6^{\circ}$ ). Next day the animal was ailing and the temperature had risen to $104.5^{\circ}$, remitting but slightly; on the third day of the fever it remained high $\left(105 \cdot 4^{\circ}\right)$ and nearly continuous, and death took place early on the fourth. The blood was quite free from specific contamination throughout the attack. At first, coagulation was slow, and small protoplasmic masses were seen; towards the end there were large granular protoplasmic masses, with plasma clear in the morning and faintly granular in the evening, with little protoplasm and few white cells, red discs shrunk and blending; no moving particles of any kind were detected.

The animal was evidently poisoned by the saliva injected, yet it did not present the same aspect as seen in spirillum fever. There was inability to sit up and the head did not droop low, but was leaned against the wall; attempts to eat were shown, which were ineffectual through debility, as it seemed, and finally, there were signs of paralysis on the left side of the body. After death, meningeal hæmorrhage was found over the right hemiphere of the brain, and there was no inflammation of the small intestines, such as I found in the body of a monkey dying on the same day of spirillum fever.

3. S. Temp. $101 \cdot 2^{\circ}$, rising next day to $104 \cdot 6^{\circ}$; the fever being continuous it was $104.8^{\circ}$ on the third day, and $105.4^{\circ}$ on the fourth. Slightly remitting, it rose to $105 \cdot 6^{\circ}$ on the fifth day, and further remitting, attained the same elevation on the sixth; on the seventh day there was a complete intermission, with an evening paroxysm $\left(105 \cdot 6^{\circ}\right)$; on the eighth a remission at first, and then an intermission (temp. $\left.101 \cdot 6^{\circ}\right)$, followed by exacerbation on the ninth $\left(105 \cdot 8^{\circ}\right)$; again a decline and rise, but now not so high (temp. $104^{\circ}$ ), and the animal sank early on the eleventh day of experiment. At first the blood resembled that of B., then it became clear and showed small clumps of protoplasm, the red discs being unchanged; on the fourth day there was 
nothing peculiar visible, white cells were few, and so next day; on the sixth sloughing of the nates on the right side had began, yet the blood was not altered in aspect; in the intervals of pyretic abatement the monkey rallied a little, but gradually became exhausted by repetition of fever and extension of the local necrosis. At the autopsy no striking lesion was apparent.

This animal also died from the effects of the inoculation, but more indirectly; probably plugging of the vessels had occurred in both animals. Any influence which the spirilla in the saliva may be supposed likely to exert when introduced into the blood was clearly overcome by other toxic agents, of which more than one was doubtless present in the very composite sputum.

Forty-third experiment.-A. patient, who had a severe attack of relapsing fever (vide No. 3) on the second day of the first interval, was rallying from the "fall" after invasion, and furnished saliva which contained epithelium, bacteria forms, granules, and débris, but no spirillum was seen. A small quantity was injected in the usual manner.

2. B. 1 , a healthy monkey, temp. $101 \cdot 8^{\circ}$. After twelve hours, fever set in, which was high $\left(105 \cdot 6^{\circ}\right)$ and of intermitting character for the first three days, and then became continuous at equal elevation for six days. On the tenth day there was a decline, followed by a sharp rebound (temp. $106^{\circ}$ ), after which the temperature slowly declined to a sub-normal level in the course of four or five days more. The blood had a dark hue; it was regularly inspected throughout this prolonged attack, and never showed any signs of the spirillum or other forms of bacteria, even after treatment with acetic acid; its characters varied only within quasi-normal limits. The animal suffered considerably, and at first the most. It became prostrate on the second day and lied down, refusing food, the body hot, and the limbs cold, and the left arm seemingly powerless. When the paroxysms ceased and pyrexia became continuous, the monkey seemed to rally, sitting up and looking about; it grew thinner and weaker, how- 
ever, and did not attempt to eat. Presently, and before fever had ceased, improvement began, and at last convalescence was prompt; the rebound did not appear to entail additional suffering; the palsy of the left arm was persistent.

By the only mishap which occurred in my experiments this animal had been injected with spirillar blood the day before inoculation with the saliva. I do not think the prior injection had any considerable influence upon the results noted, which were similar in character to those of the preceding experiment, No. 42. B. 1 was shortly afterwards again inoculated with spirillar blood, and the successful result is described under Experiment 8.

Forty-fourth experiment.-The saliva of a perfectly healthy man contained epithelial scales, micrococci, bacteria, vibrios, and granular matter; spirilla were not detected in the fresh state, but after treatment with acetic acid, wavy filaments were seen, which had all the aspects of them. Ten minims of this fluid were injected in the usual manner and place.

2. B. B., a monkey in healthy condition, though a few weeks before the subject of spirillum fever, showed on the day following some inflammation of the thigh, and a small abscess at the site of injection three days later. There was no fever (maximum temperature on fourth day, $103 \cdot 2^{\circ}$ ), or only a slight exaggeration of the normal daily cycle, with a tendency to decline below the mean, and the blood was unchanged on the first four days ; convalescence was complete.

The above experiments indicate the presence of a toxic agent in saliva which is not the spirillar, and should the latter exist, it seems to be overcome by the former, what. ever its nature, Healthy saliva is irritant, and during and after the spirillum fever this quality is greatly intensified, so that even death may ensue from the inoculation of febrile sputum. Germs of the mouth-parasite are not developed within the blood of an animal susceptible of direct contamination from the blood of another infected animal. 


\section{CHARTS}

Illustrating Dr. V. Carter's Inoculation Experiments.

Series 1.-Relapsing Fever (spirillum fever) in Man.

(In Diagrams I to IV.)

The temperatures are axillary and shown in the usual manner.

The state of the blood is indicated by the signs + and -, signifying respectively the presence and the absence of the spirillum at the time of examination.

By the sign \pm is meant the apparent absence of the parasite in fresh blood, and its subsequent detection after drying of the specimen and treatment with acetic acid.

N.B.-These charts will also serve to show the identity of the fever as seen in Bombay, with its European forms.

\section{SerIEs 2.-Artificially induced Fever in the Monkey.}

\section{(In Diagrams V to VIII.)}

The temperatures are axillary, and are given for every three hours of the day.

The state of the blood is shown by the signs + and - , which respectively signify the presence and the absence of the spirillum at the time of examination.

The sign \pm means spirillum not seen in fresh blood, but seen after drying and the application of strong acetic acid (Albrecht's process).

$P$ Indicates appearances not wholly affirmative.

N.B.-The dotted line immediately above that of $101^{\circ} \mathrm{F}$., represents the mean normal temperature, viz. $101 \cdot 3^{\circ} \mathrm{F}$. 
Med Chir Trans Vol LXII. Diriogram E-VIIIT) 120.

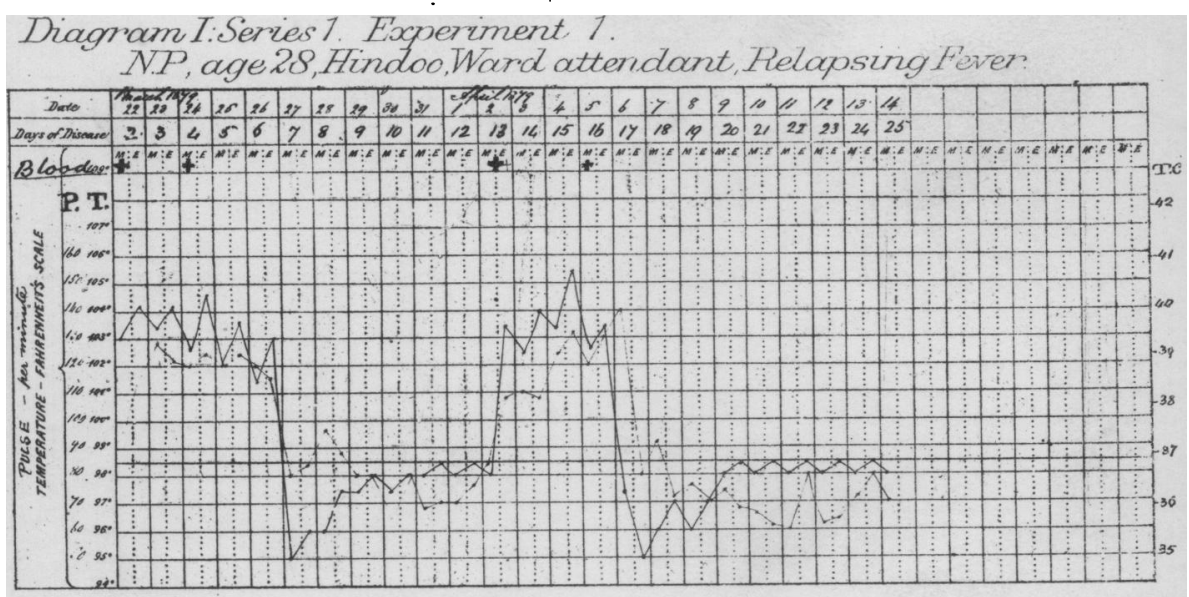

\section{Fixperiment 2 .}

IIS fernate, age 60 Mrussutman, Beggar Relapsing Fever:

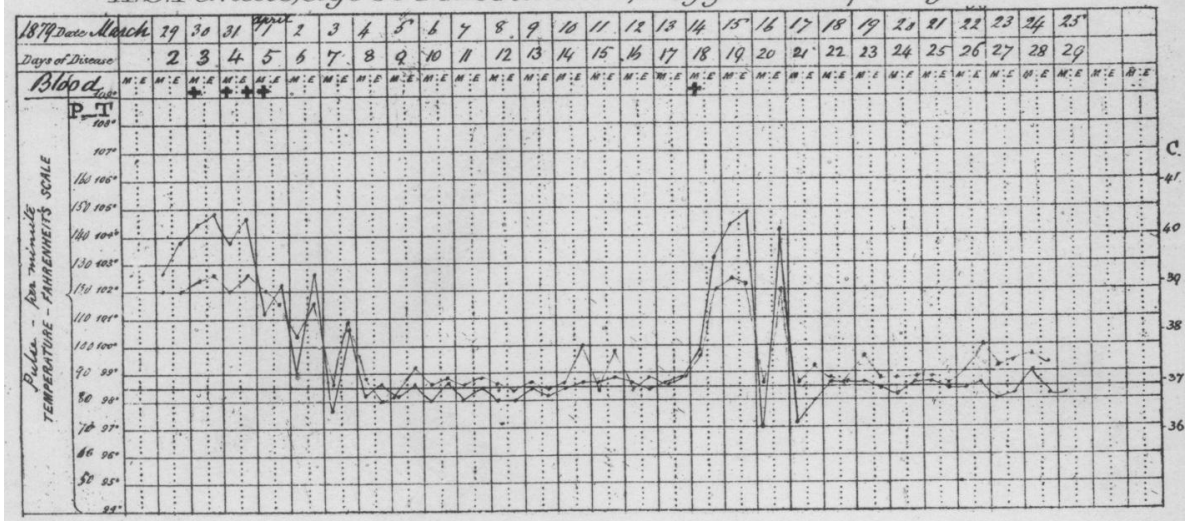

\section{Experiments 3,11, 43 .}

E.S.age 25, Mussutman, Coal Trimmer Relapsing Fever.

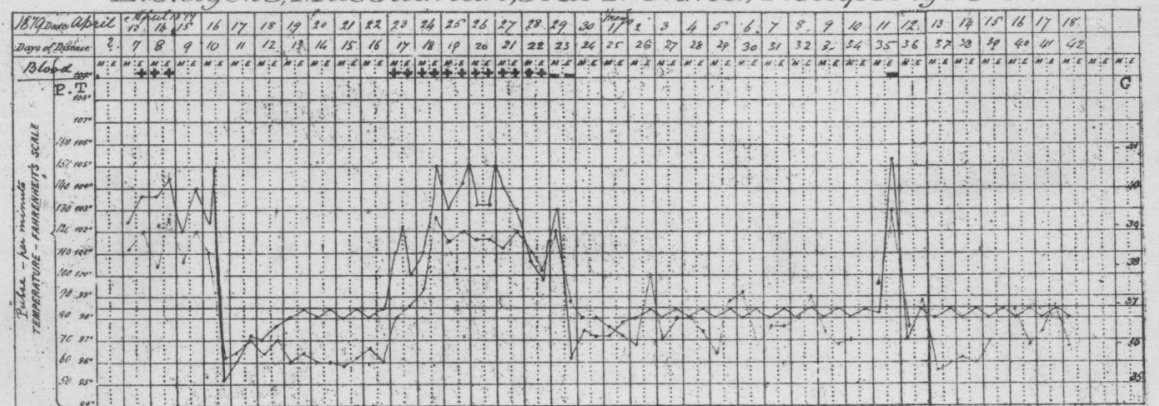


Thagraun II: Serzes 1. Experiments 4, 20

H.T.uge 22 Hindoo, Municipal Sweeper, Retapsing Fever.

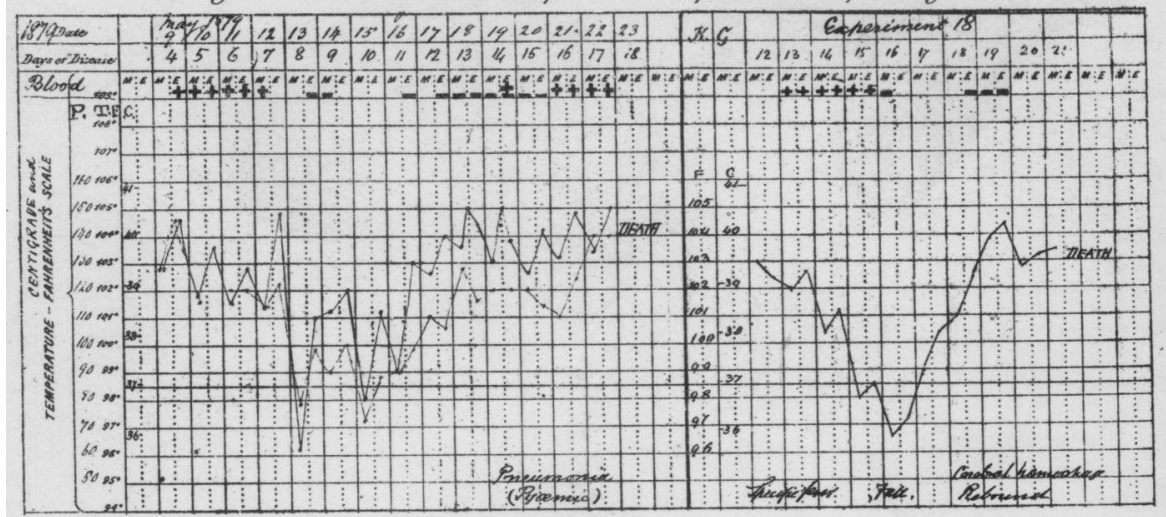

Expueriments 5,19.

B.Mage30, Mussutman, ShipLabourer Relapsing Fever:

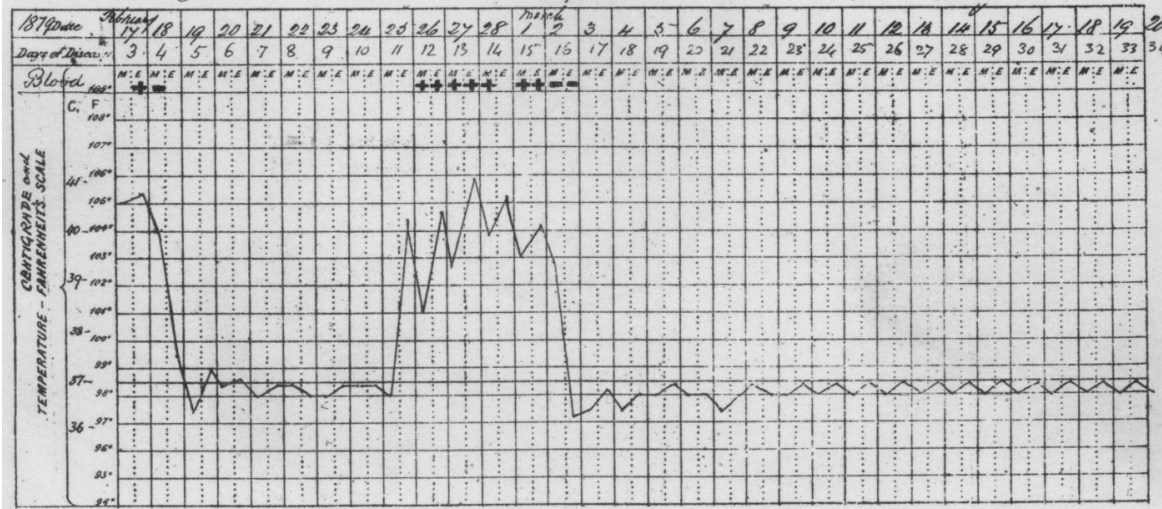

\section{Experiment 6}

I.S.B.age14, Mussulman, Coolie Labourer Retapsing Fever.

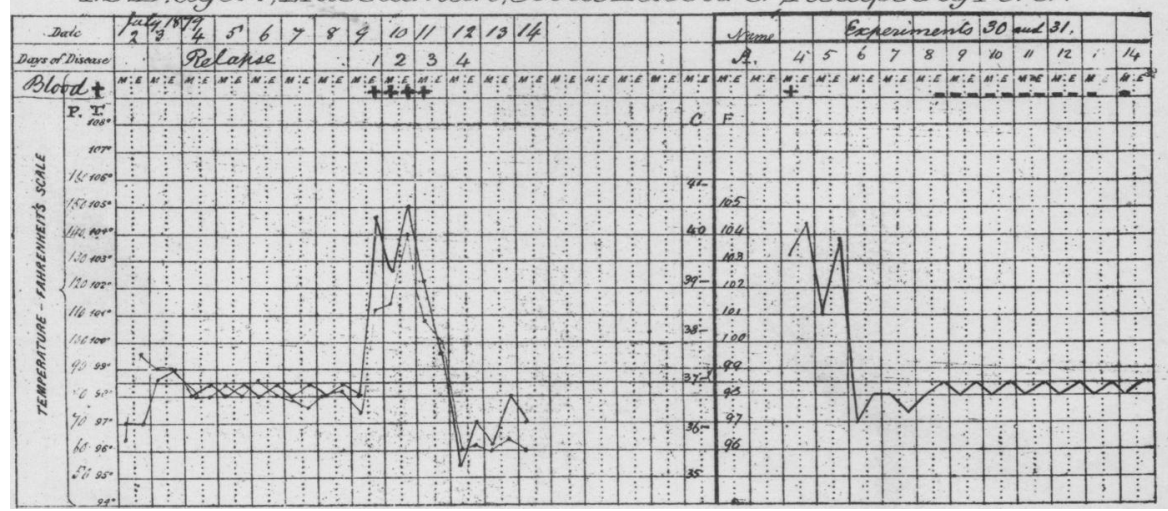


Diagram III:Series 7. Experiment 7.

B. age 34. Hindoc, Coctie Labouner, Famine Immigrant, Relapsing Fever:

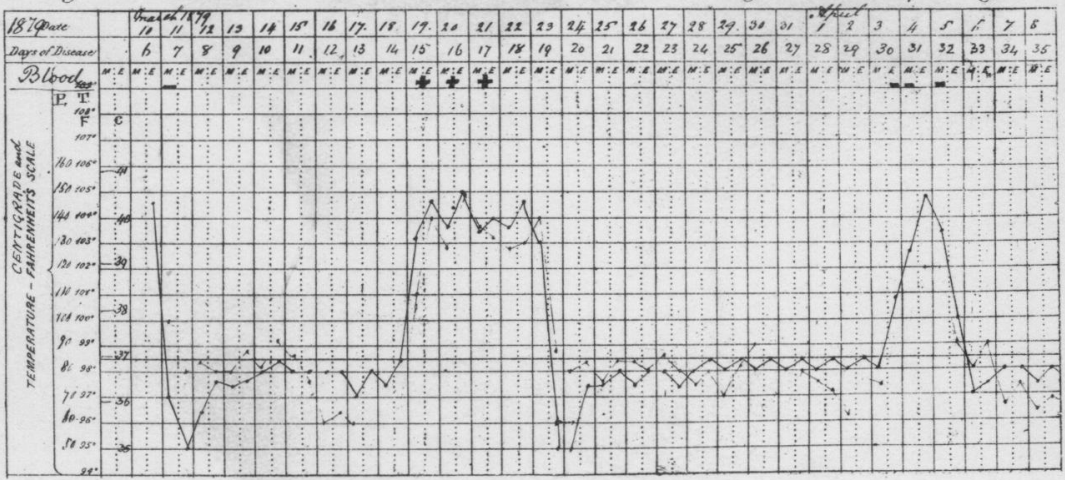

\section{Experiment 7. continued}

\begin{tabular}{|c|c|c|c|c|c|c|c|c|c|c|c|c|c|c|c|c|c|c|c|c|c|c|c|c|c|}
\hline & 1011 & 12 & 19 & 14 & 15 & 161 & 718 & & 20 & 21 & 22 & & 24 & & & & & & & 12 & & & & & \\
\hline 36 & \begin{tabular}{l|l|l}
37 & 38 \\
\end{tabular} & 39 & 40 & 41 & 42 & $43 \sqrt{4}$ & & & 47 & 48 & 49 & 50 & s7 & 52 & 53 & & & & 58 & 59 & 60 & & & & 6566 \\
\hline & & & & & & & & & & & & & $\xi$ & & & & & & & & & & & & \\
\hline & & & & & & & & & & & & & & & & & & & & & & & & & \\
\hline & & & & & & & & & & & & & & & & & & & & & & & & & \\
\hline & & & & & & & & & & & & & & & & & & & & & & & & & \\
\hline & & & & & & & & & : & & & & & & & & & & & & & & & & \\
\hline & & & & & & & & & & & & 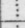 & & & & & & & & & & & & & \\
\hline & & & & & & & & & & & & & & & & & & & & & & & & & \\
\hline & & & & & & & & & & & & & & & & & & & & & & & & & \\
\hline & & & & & & & & & $\triangle$. & & & & & & & & & 3 & & & & & & & \\
\hline & & & & & & & & & & & & & & & & & & & & & & & & & \\
\hline & & & [: & $1:$ & & & & $t$ & & & & & 1 & & & & & & & & & & & & \\
\hline & & & & & & & & & & & & & & & & & & & & & & & & & \\
\hline & & & & & & & & & & & & & & & & & & & & & & & & & \\
\hline
\end{tabular}

\section{Experiment of}

F. T. age 28, Mussulmaz, Ship Labour er: Relopsing Fever:

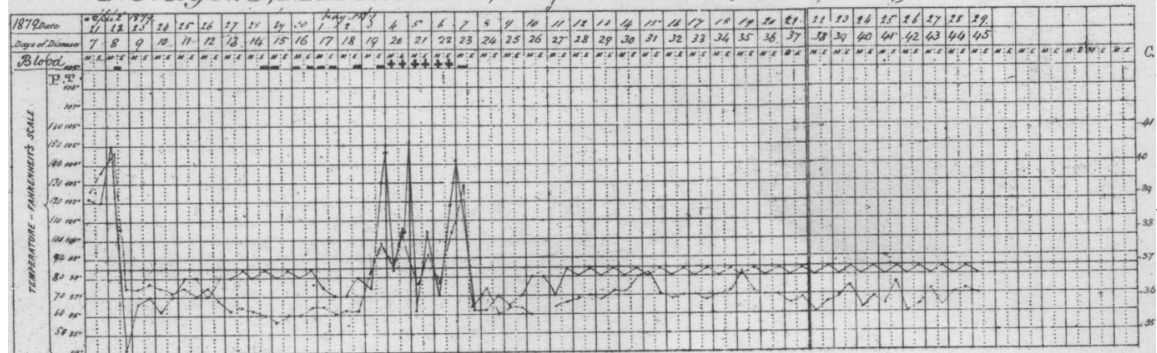




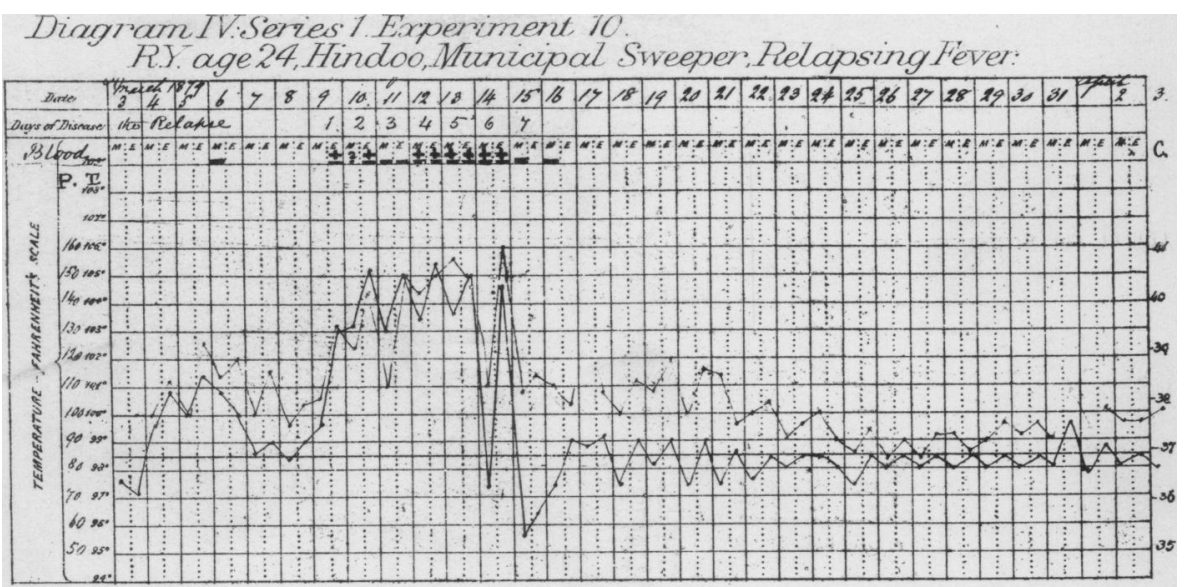

\section{Experiments 9, 13, 21,22,27,32,33,34.}

SB.SP age 45, Mussutman, Weaver, Retapsing Fever:

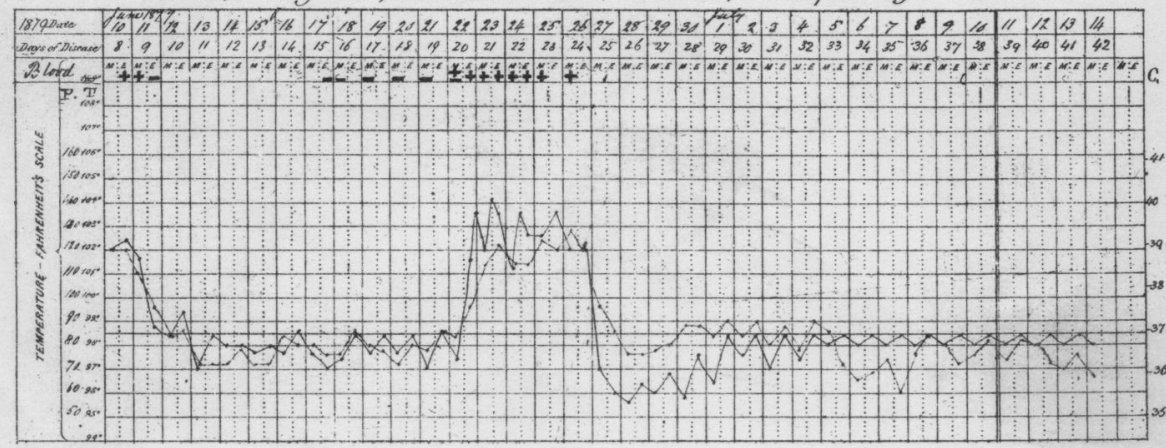

\section{Experiment 72}

AMage 30, Mussulman, Labourer. Fetapsing Fever:

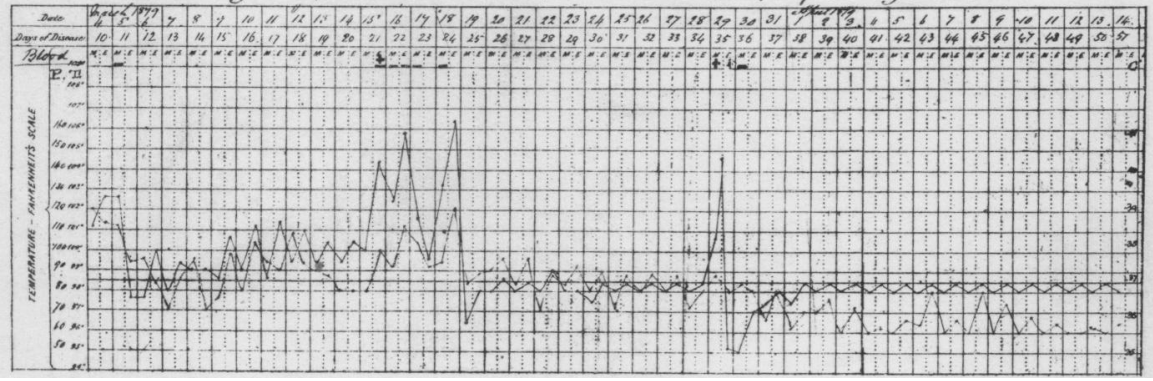


Diagram V. Series' 2. Experiment 1, 2. Monkey GP,WB, \&WF.
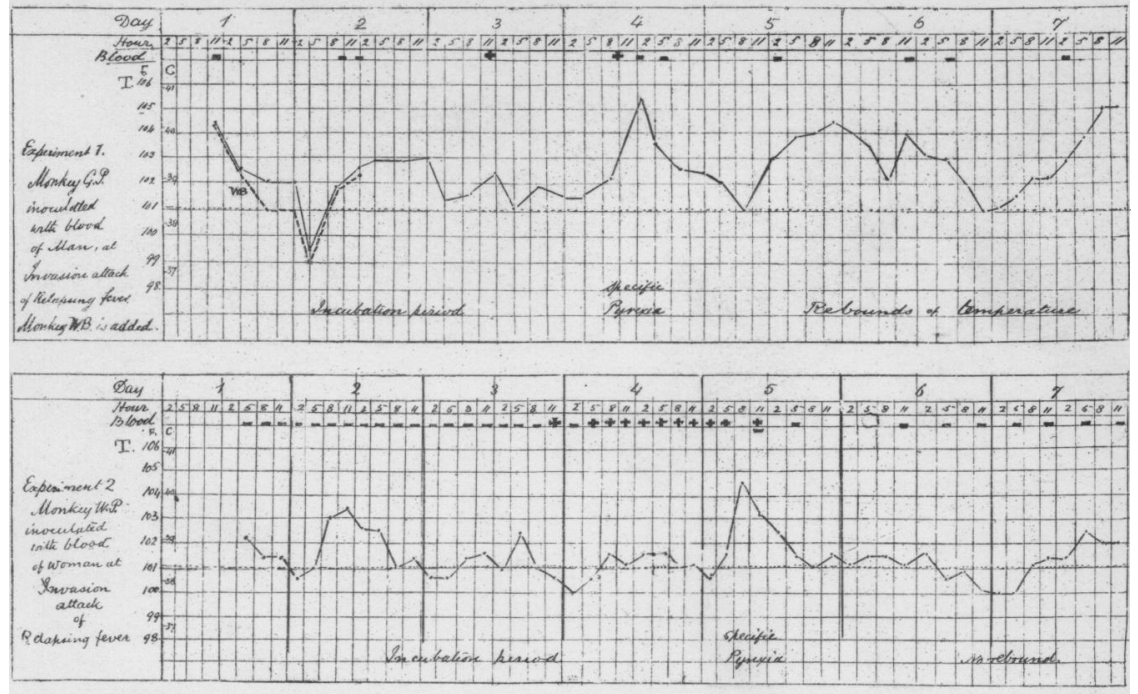

Experiment 3, 7,11, Monkey, B, 3, YT, \&WT.2.
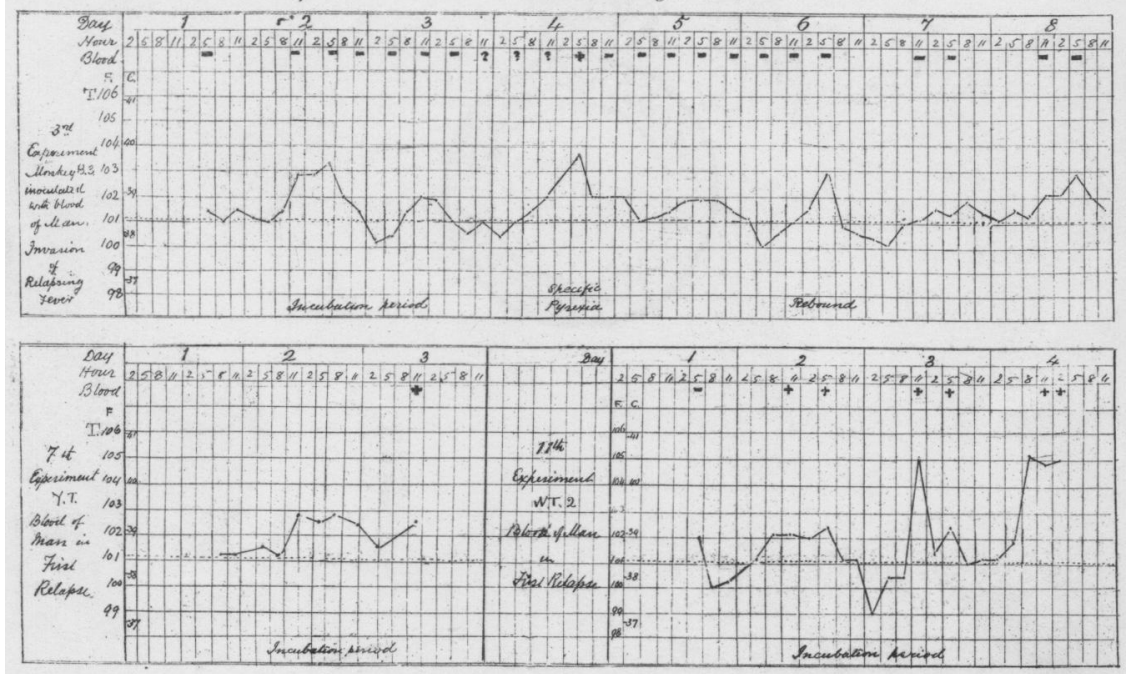

Experiment 6, Monkey, I, Chain.

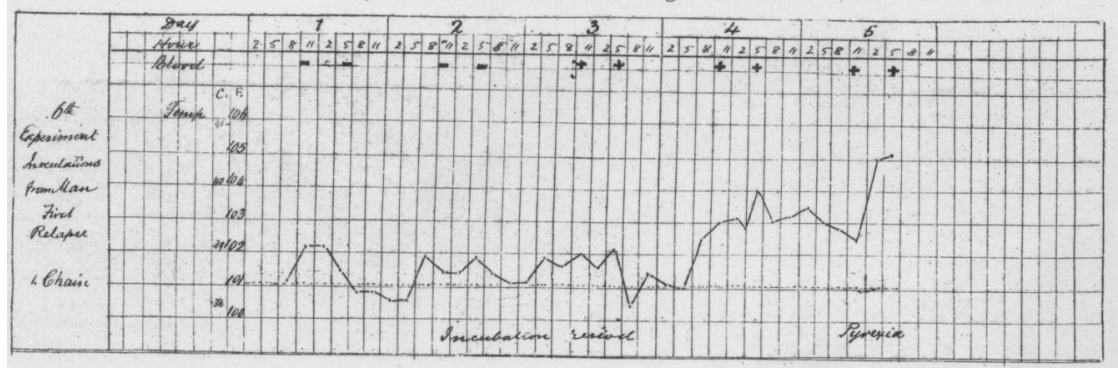


Diagram VI; Series 2. Experiment 2, Rope, 9,WT,3

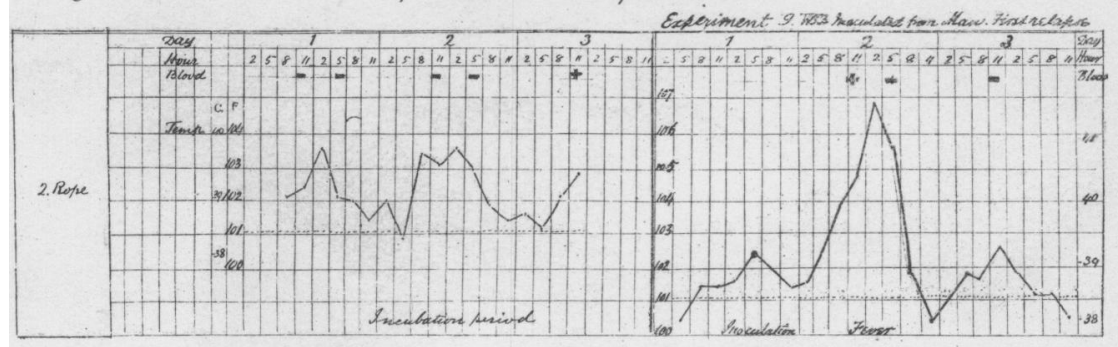

Experiment 8, BI, W,WP
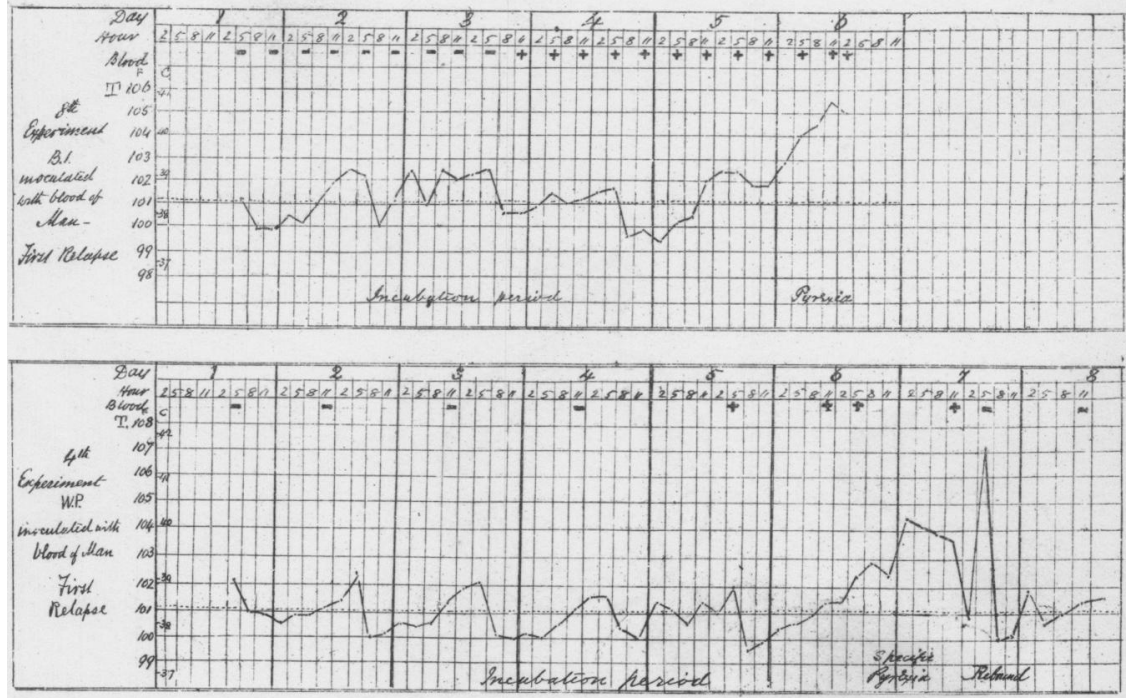

\section{Experiment $12, B B$}

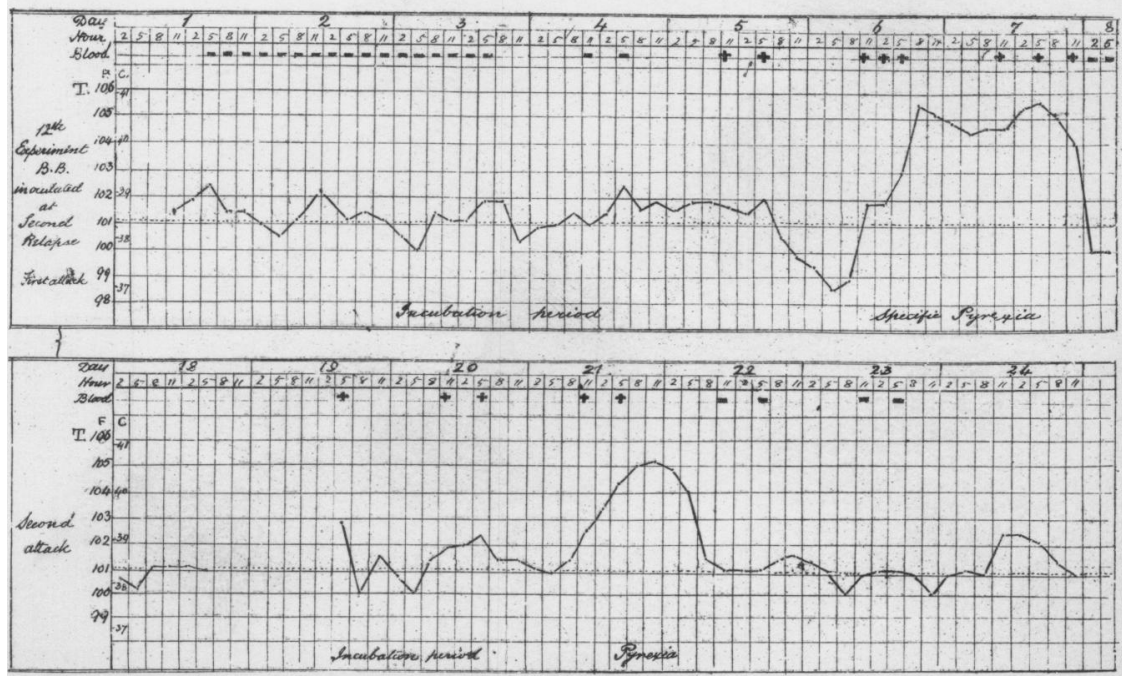


Diagram VII: Series 2. Experiment 14,RT, 17, E
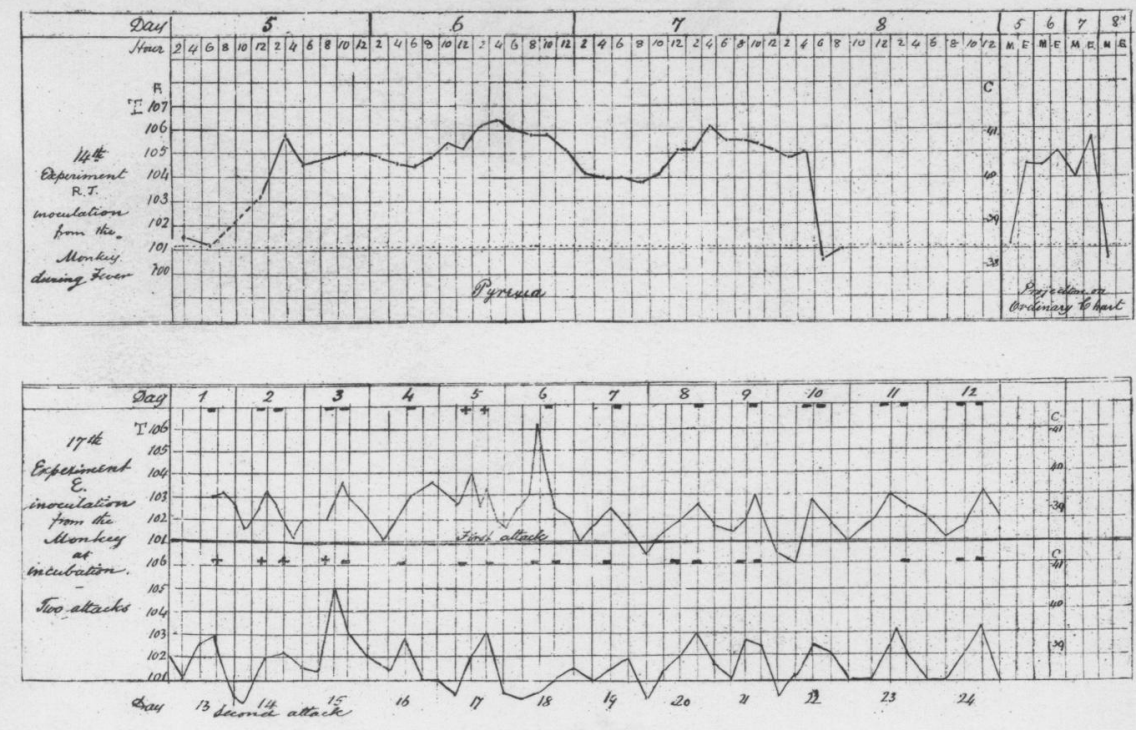

Experiment.15. N,W,T,R, C.

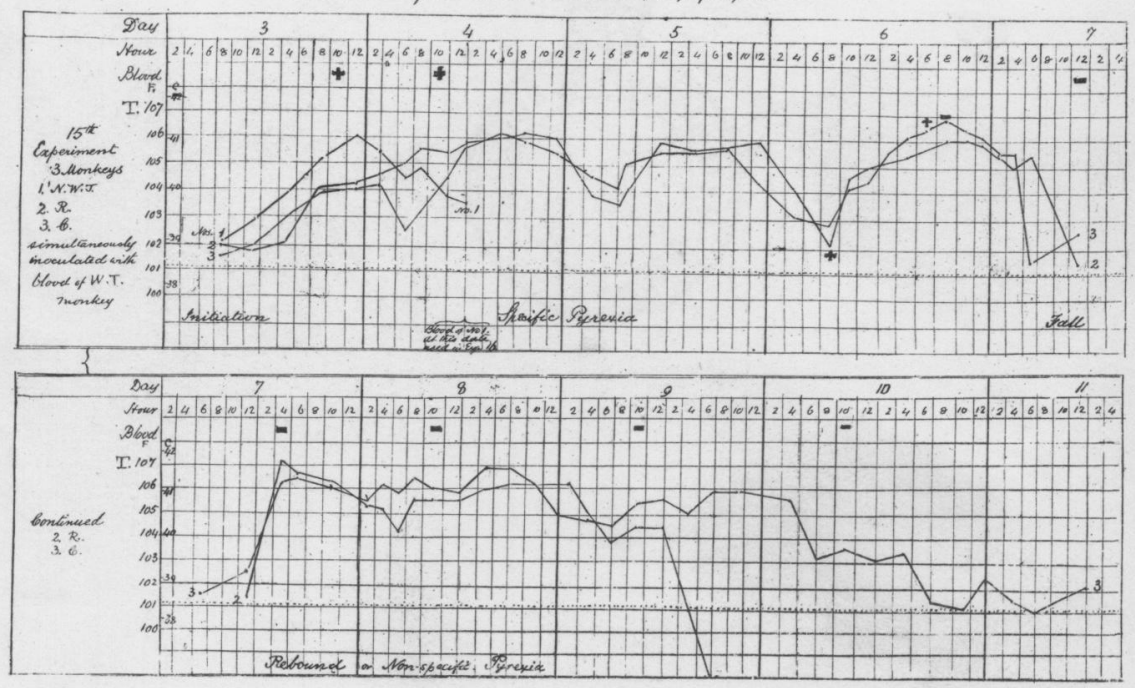

Experiment, 16, BT, \& OT:

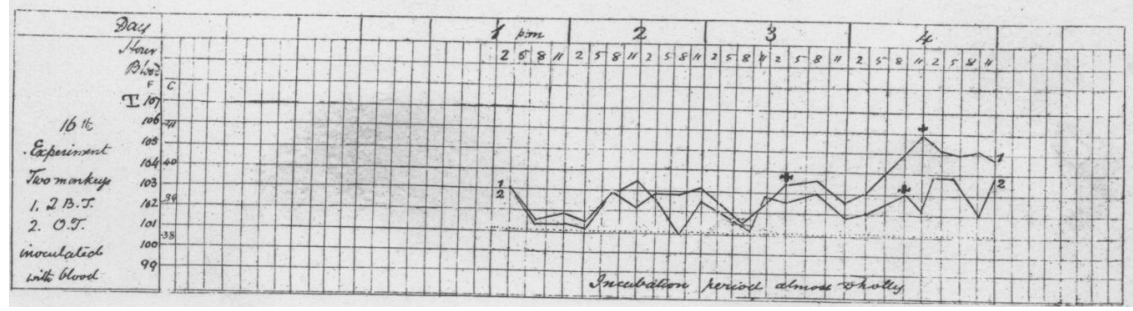


Diagram VIIISeries 2. Fapperiment 16, contimued.

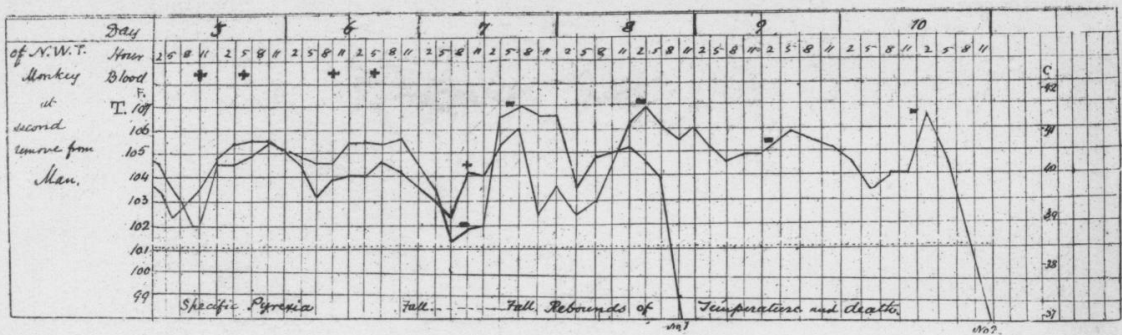

Fixperiment 42.B, $S ; 43, B 1$
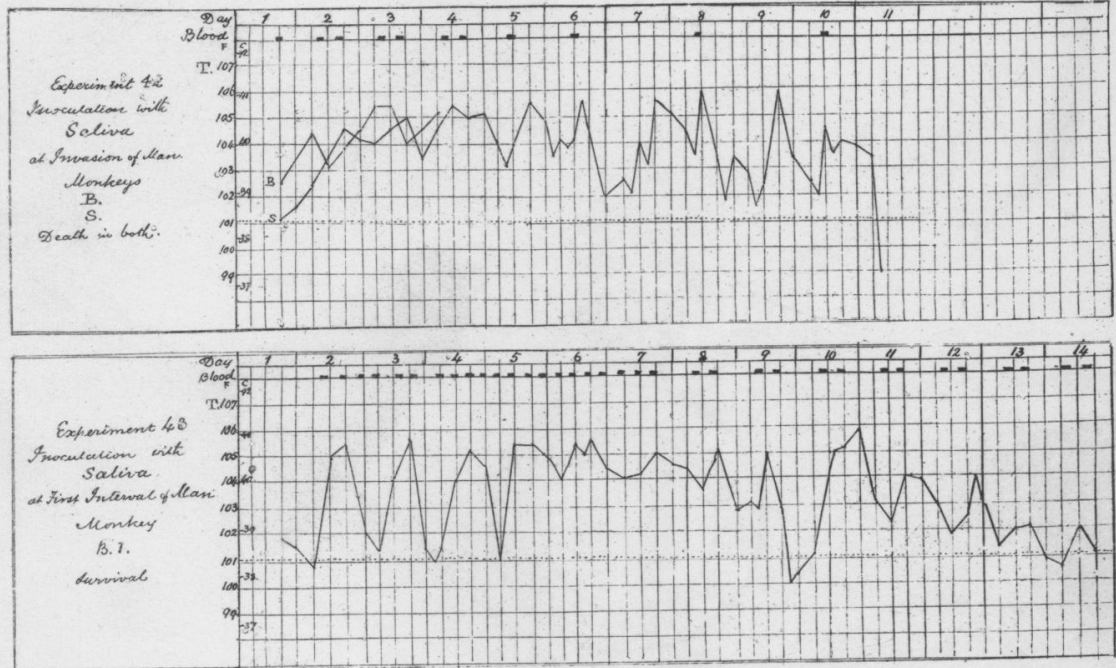

Appendixe.

Monkey $7 \& 2$.
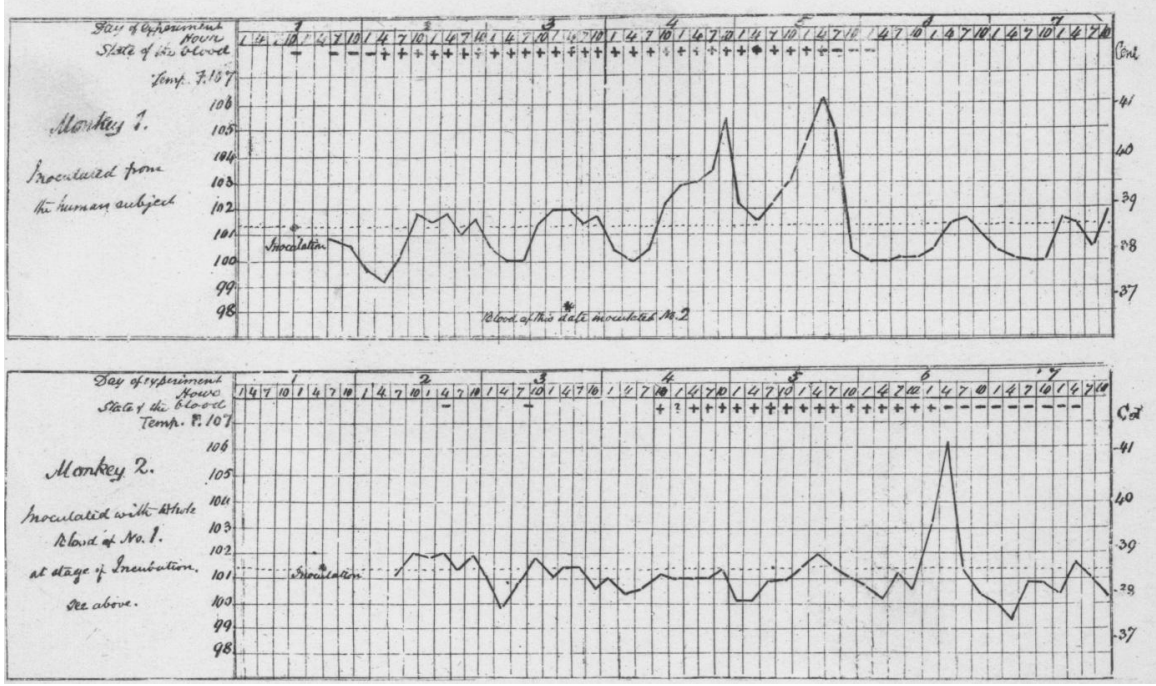


\section{INDEX TO EXPERIMENTS.}

A. WITH FRESH SPIRILLAR BLOOD. Series 1.-Positive results. Inoculations from Man.

EXP. STAGR OF PEVER. PAGE

1. Invasion . 4th day (2 inocs.) 82

2. $"$ 4th $"$. . 83

3. " 8th " . . 84

4. 1st relapse, 1st " . . 85

5. $\quad$ 2nd " . . 86

6. " 2nd " (2inocs.) 88

7. $"$ 3rd " . . 89

8. $\quad$ 3rd,$\quad . \quad$. 89

$9 . \quad$ 3rd $” \quad . \quad .90$

$10 . \quad$ 4th $"$. . 91

$11 . \quad 4$ th $" . \quad 92$

12. 2nd " 1st " . . 94

13. 1st interval, 9th ” . . 95

Inoculation from the Monkey.

14. 1st day of attack . . 97

15. 2nd $" \quad(3$ inocs.) 98

16. 2nd $\quad$ (2 inocs.) 101

17. 1st day of incubation . . 103

Series 2.-Negative results.

From Man.

18. Invasion at decline . 105

19. 1st relapse, $3 \mathrm{rd}$ day ( 2 inocs.) 106

20.

" 1st , a.m. . 108

22. " 1st ” p.m. . 108

From the Monkey.

23. Incipient pyrexia $\quad . \quad 109$

24. Incubation . . . . 109

25. $\quad$ end . . . 109
B. With NON-SPIRIIILAR BLOOD.

Series 3.

EXP. STAGR OP FEVYR. PAGE

26. Incubation (Monkey) . 110

27. Fall (Man) . . . . 111

28. " (Monkey) . . . . 111

29. Rebound (Monkey) . $\quad 112$

30. 1st interval (Man) . . 113

31. $" \quad " \quad .113$

32. $"$ " 113

$33 . \quad " \quad 0 \quad 113$

34. $"$ " $\quad$. 113

C. WITH DRIED BLOOD.

Series 4.

35. Fall (Man) . . . . 115

36. 1st relapse (Man) . . 115

37. Specific fever (Monkey) . 115

38. 1st relapse (Man) . . 115

39. Specific fever (Monkey) . 115

40. 1st relapse (Man) . . 116

41. Specific fever (Monkey) . 116

D. With saliva.

Series 5.

42. Invasion (Man), 2 inocs. .116

43. 1st interval (Man) . . 118

44. Normal (Man) . . . 119 


\section{PART II.-COMMENTARY.}

The above forty-four experiments were made with material derived from man (in thirty-one instances) and from the monkey (in thirteen), upon fifty-one of the lower animals. Serious attempts upon the human subject become unnecessary in the light afforded by my comparative data, for I presume there is no doubt that the blood of an individual suffering from relapsing fever is capable of communicating the same disease to an unaffected subject, just as it does to the healthy quadrumana, and also to these animals amongst themselves.

The chief group embraces the essays made with blood containing the spirillum. Of these there were twenty-one inoculations from man with six failures (i.e. 1 to 3.5 ), and ten from the monkey with three failures (i.e. 1 to $3 \cdot 3$ ), or more properly nine with two; the total is thirty-one with nine failures. Even thus crudely stated, the preponderance of successful inoculations is so decided as to render it certain that the spirillum fever is even readily communicated from man to the lower animals, and between the latter themselves; and this being so, the exceptions to the rule claim special notice, not only from their intrinsic interest, but from that bearing upon a series of complementary experiments, which were made with a view of testing the positive results. The significance of this further series of nineteen essays giving negative results, would be much modified were it established that infective material was commonly or even frequently inoperative, and I will, therefore, at once point out the circumstances under which the somewhat unlooked-for failures occurred, with the inferences that may hence be drawn. 


\section{A. Negative Series.}

Remarks on the second series, Nos. 18 to 25 inclusive.Whilst under most conditions spirillar blood is capable of conveying infection, the following states seem unfavorable to this result :-A period prior to the onset of fever (No. 25), or at the very beginning of high fever (Nos. 21, 22, 23), when I may here observe the parasite will not grow readily under artificial cultivation; again, at the acme of attack with high temperatures (No. 19), or at the very end (No. 18), when also cultivation fails. The present series shows, too, that when pyæmia is present in conjunction with spirillar infection, the blood conveys not the latter, but a contamination possibly like the former (No. 20), and, finally, if a second poison (e.g. of saliva) be injected immediately after the spirillar, it overcomes or annuls the latter (No. 24). Upon review, therefore, these anomalous instances appear not inexplicable, and, further, they become highly suggestive.

Remarks on some other discrepancies.-Experiment 17 showed that blood at early or non-spirillar incubation stage may convey infection, whilst No. 26 displayed only negative results in a similar trial. There is also to note the discordance of Experiment 13 with Nos. 32, 33, and 34, for as they stand these data show that the blood of man and animal, during the ante-febrile stage, may or may not convey infection. When all the evidence is summed up, however, the balance is decidedly against the likelihood of the non-febrile incubative period being an infective one ; and of the two instances to the contrary just named, in one (No. 13) there seemed an inferential probability of contagion, and in the other (No. 17) the mon. key appeared unusually susceptible, for it presented a "relapse," and this, at least, may have been incidental. I would further remark that the advent of the spirillum during the pre febrile stage being intermittent, it seems 
possible that an incubation experiment may succeed at one hour and not at another during this period.

Remarks on contagion as a disturbing influence in these experiments.-The possibility of an affected animal communicating its disease to another by means of contact was early recognised, and I made some trials to test this with negative results. Contagion was never actually proved, in so far that no animal not operated on ever showed the specific fever; possibly the hairy integument was a protection more or less complete, yet bearing in mind the case in man, I do not deny that amongst the lower animals contagion may have occurred in spite of some measures taken to prevent it, and it is not impossible that the anomalous instances of Nos. 9 (quasi-immediate infection), 13 (infection at first interval), 12, and 17 (relapses), may owe their peculiar features to that agency ; for this reason, which is only inferential, they will not be insisted on in the final summary. In the great majority of successful inoculations there was so much concordance in time, that I do not hesitate to exclude the influence of contagion. Whether or not the monkey could acquire the fever by simple contact with human patients is not known; none of the persons handling the sick animals were attacked.

Remarks on the negative Series 3 and 4.-In Series 3 are nine experiments with non-spirillar blood, showing no ill effects, namely, one with blood at period of incubation (No. 26), two with blood at or just after the "fall " (Nos. 27 and 28), one with blood of " rebound" (No. 29) ; also two with blood of man in "first interval" when no relapse occurred (Nos. 30 and 31), and three with human blood in "first interval with relapse" following (Nos. 32 33 , and 34). Upon consideration I am disposed to consider these instances as valid evidence, that in the absence of the blood-parasite inoculations fail; and I add that under the test of artificial cultivation the same non-spirillar blood never evolved the specific parasite.

The negative effect of inoculation with dried blood 
taken during fever or at the fall, I am disposed to consider as fully settled; and some other means than desicca. tion is needed to preserve intact the contagious property of infected blood. This bears on the rôle of fomites.

As regards Series 5, it seems equally obvious that the spirillum in the sputum does not, upon injection of the saliva entire, induce the specific fever; whether or not it would do so in an isolated state cannot well be known. The different effects of febrile and non-febrile saliva, though structurally alike in abundance of bacteria, \&c., is worthy of notice.

In bringing these preliminary remarks to a close, I will only observe that the discordant results elicited in my inquiries produced some surprise until it was considered that the propagation of infective diseases in man is by no means uniform, invariable, or comprehensible as regards exceptions to the rule. These experiments show that (like ordinary typhus) the spirillum fever is not conveyed at all stages or upon all occasions, and they point to some of the exceptional conditions, which I need not recapitulate. Supposing that the contagious element is represented by the spirillum, I find grounds for believing that the parasite is not equally active at all periods of the fever, and not at all so during the ante-febrile state; the discrepancies, indeed, in my experiments are marked enough to render it doubtful if the spirillum itself does represent the contagium proper, and not rather some other agency which at certain periods is associated with it. Such doubt seems warranted so far as regards the earlier scanty proportion of the parasite, its dynamical properties at high temperatures or immediately preceding its total disappearance at the "crisis," and lastly, when associated with some other blood poisons (also possibly parasitic).

Additional inferences might be drawn from the above negative series as regards, for instance, the condition of the animals operated on, which will be suggested by the experiments themselves, and there are, besides, several important points not even alluded to; but I claim for my 
work no more completeness than commonly attends a first essay, especially as regards negations which are difficult of proof, and the following series is, perhaps, the more satisfactory.

\section{Positive Series.}

The data I have to offer concern the changes of temperature and state of the blood consequent upon spirillar infection artificially induced; a few remarks upon the post-mortem appearances at different periods of infection are added, and some collateral evidence derived from culture-trials.

Memorandum on the normal temperature of the monkey.It is necessary to be aware that these small creatures have a greater body-heat than man. The axillary temperature of four healthy animals was taken in the usual manner, and under common conditions, at three-hour intervals during from two to five days; total observations 97 ; mean temperature of air $80^{\circ} \mathrm{Fahr}$. The data obtained were at least as uniform as have been stated for man, and are summed up as follows :

\begin{tabular}{|c|c|c|c|c|c|}
\hline \multicolumn{3}{|c|}{ Daily mean temperature } & & • & $101 \cdot 35^{\circ} \mathrm{F}$. \\
\hline Mean dail & ange & . & & • & - $100.4^{\circ}$ to $102.3^{\circ}$ \\
\hline Maximum & • & . & • & • & . $103^{\circ}$ \\
\hline Minimum & • & . & . & $\bullet$ & . $100^{\circ}$ \\
\hline
\end{tabular}

The daily mean temperature is nearly the temperature at the hours of 7 a.m. and 7 p.m.; the maximum was found at $5-6$ p.m., the minimum at $10-11$ p.m.

Course : the lowest temperature occurs about midnight $\left(100 \cdot 5^{\circ}\right)$; a gradual rise takes place after 2 a.m., continuing till the afternoon, when ( 5 p.m.) the highest point is reached $\left(102 \cdot 3^{\circ}\right)$; then follows the evening depression, which proceeds more rapidly than the morning rise.

As compared with man the mean temperature is nearly $3^{\circ}$ higher; the range of $1.9^{\circ}$ is about the same, and the course followed throughout the day is very similar. The 
Normal Temperature of Monkey.

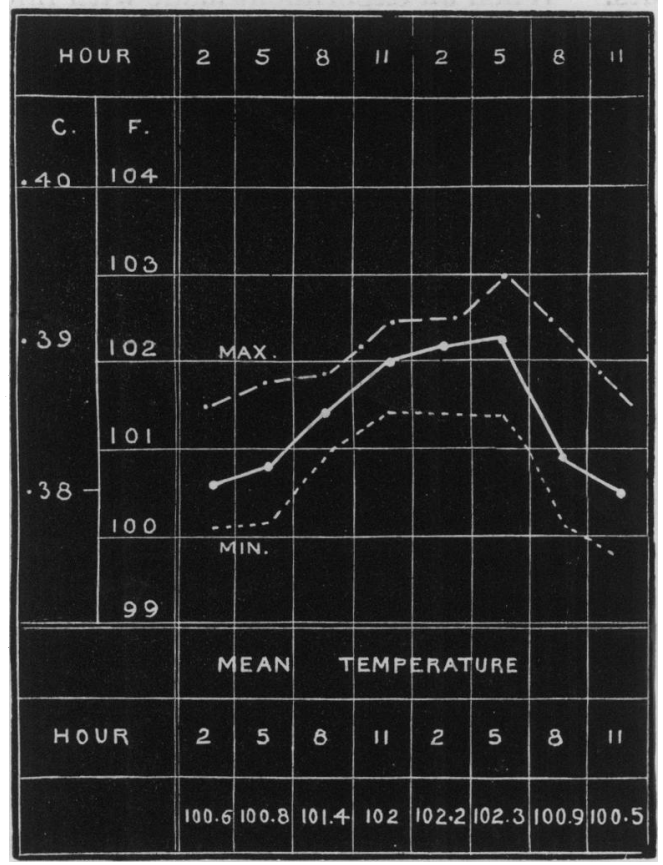

mean daily temperature of healthy natives, taken in the usual manner, I find to vary in range, and oftenest to be rather below that of Englishmen at home.

Normal variations. - The data show these to be not very considerable. They consist in tendencies to a narrower range, to a slight rise soon after midnight, and to an elevation preliminary to the common mean maximum near the hour of sunset.

Incidental variations. - A temporary exacerbation follows restiveness or excessive muscular exertion; and the presence of sores due to biting, with chafing of the band or chain holding the animal, also leads to perturbations of temperature. In cool and damp weather the body heat declines, especially at night.

Some quasi-normal temperatures.-Without entering 
into details, I would here allude to my records of abortive experiments. When an experiment made with infecting or non-infecting material fails to produce an effect on the monkey, the temperature retains nearly or mostly its normal characters, and the same is true for the period immediately following an ordinary spirillar attack. This statement is not, however, exhaustive; for although I found that desiccated blood and the blood during the incubation period, at "fall," "rebound" and at date of expected relapse of man, did not induce decided constitutional disturbance, as measured by the bodily heat, yet some perturbations of temperature may ensue, and it has still to be positively determined that these do not represent the mildest or quasi-abortive developments of the blood spirillum. In my observations even at three-hour intervals some minute or very brief blood changes, really specific, may have been overlooked which would correspond to these temperature perturbations when there was no other apparent influence at work; and it would be highly significant, if the spirillar infection, in whatever degree, was established under any of the conditions named above. The chief obstacle to advance in this direction is the want of definite knowledge regarding the development of the spirillum itself, by which to interpret such blood changes as are not seldom seen; and I have many times reluctantly recorded a negative observation owing to this ignorance.

General conditions of experiments. -70 per cent. of my essays, including the first and last that were made, proved successful ; and I am of opinion that, with the precautions above intimated as necessary, a much larger proportion of successes might be obtained. In the seventeen trials noted below, twenty-two monkeys were employed, and I find that when two or three were used in a single experiment the result was sufficiently alike, as regards both time and degree, to exclude the idea of contagion as an intervening influence; and as regards the occasions when the same animal was at successive intervals subjected to 
repeated inoculations, I had no reason to conclude that the earlier essay materially influenced the later ones. The prime source of infection was the blood of human patients at first, second, or third attack of relapsing fever of ordinary type; subsequently blood inoculations were made from one animal to another.

The induced fever in the monkey can here be compared only in part with its human prototype; yet I am able to say that there was throughout a degree of concordance in general symptoms of oppressive character, which clearly indicated that the "fever" was virtually the same. Postmortem appearances correspond in the main.

\section{Observations on the Pyrexia.}

Its general features.-The attack in the monkey being initial is to be compared with the first or invasion attack of man, which in him has a strikingly uniform duration, course, and intensity, and in three fourths of cases is followed by a relapse. In the lower animal there is much more variety of form, the date of onset also varies, and a relapse is so rare (viz. at most as one in eight) and so irregular, as to appear altogether incidental or possibly wanting. No such foretelling of events is practicable here as is feasible in the human subject.

These results were not anticipated, and upon reflection I observe, as regards the monkey, that the spirillar infection being new to the species a greater range of susceptibility was likely; besides, the inoculated material was not always the same in character or amount; it may have become modified in transmission, and it was implanted in an artificial manner in healthy subjects.

The human subject, on the other hand, seems to have become uniformly susceptible to attack, is infected in a "natural," possibly uniform, manner, or at certain stages only of the fever; commonly, too, the frame is at the time predisposed by a departure from the normal state.

vol. LXIII. 
It is the same thing to say that the contagium itself may have become modified in its passage through the human system; its specific characters may even be due to gradual evolution within the same sphere.

According to my observations the relapse is an acquisition in man, and not an essential part of the fever.

\section{Particular Features of the Pyrexia.}

Incubation-period.-This is commonly regarded as a breeding time, ending in maturity of the pyrogenic agency, and reckoned as lasting from infection to fever, general symptoms being seldom well defined until towards its close, when the prodromata appear. My experiments serve to illustrate the varied duration and form, and probable nature of this period, especially as regards the nearer pre-febrile stage corresponding in date to the premonitory symptoms.

I should here remark that, correctly speaking, the relapsing fever of man is a composite affection embracing a series of distinct attacks, and therefore cannot, except in a very restricted sense, be classed as one of the "continued" fevers. The corresponding affection in the monkey, on the other hand, comprises but a single febrile event, which is probably the fundamental, as it is the simpler, manifestation of spirillar pyrexia.

Judging from analogy, the several apyretic intervals of the recurring variety should be regarded as incubationperiods belonging to the attack which they preoede, and the accuracy of this view is confirmed by the fact that the specific blood-contamination invariably commences one or two days (at least) before the onset of fever in man and animal, that is to say, towards the close of the apyretic incubation-period; whilst it is never prolonged beyond the pyrexial attack, but promptly and wholly ceases with it. Particular scrutiny is needed to detect the spirillum during the incipient apyretic infection of 
man, and in him data are necessarily wanting for the first incubation-period, or that preceding the invasionattack; but in the lower animal this preliminary bloodinfection, besides beginning earlier, is more readily detected during the first and sole incubation-stage here witnessed, and thus, by experiment, the whole series of observations is rendered complete.

Each of the non-febrile periods in question thus becomes divisible into an earlier incubation-stage proper, and a later stage of apyretic infection, during which there is present the same visible blood-contamination as characterises the pyrexial condition, the only difference being that, with the onset of fever, the parasite becomes more abundant. I was not able to correlate in the monkey any premonitory symptoms beyond occasional languor and impaired appetite with the preliminary infection stage, nor have I yet in man observed further concurrence; future inquiry may show such other coincidences as might reasonably be anticipated.

The following details of temperature are available from thirteen selected experiments :

Non-spirillar stage.-After incidental excitement has passed off there occurs but slight perturbation of body heat, the tendency in prolonged instances being to a decline, connected with effacement of normal daily range. The mean duration was 60 hours, which is probably in excess of the actual, but such wide variation is apparent (viz. from 15 to 96 hours), that it becomes evident there were conditions differently influencing each group of experiments; these might be concerned with the quantity of injected material (though hardly likely so), or with the stage of fever, or state of the blood inoculated, and of this the number and activity of the spirillum may not be a complete measure. In general, this stage was twice or thrice as long as the succeeding one.

Stage of spirillar infection.-The assertained mean temperature at first appearance of the parasite was $102^{\circ}$, or so completely within normal limits as to show conclu- 
sively that the mere presence of the spirillum does not entail high fever; subsequently the body heat even declines from $\cdot 3^{\circ}$ to $\cdot 9^{\circ}$ below the normal mean of corresponding hours at day-rise especially, and if this stage be prolonged the temperature may sink to $98 \cdot 6^{\circ}$, or below the normal minimum. I have verified this course in the human subject. The mean temperature immediately prior to the estimated hour of febrile onset was $101 \cdot 6^{\circ}$, or hardly above the normal mean; once it was $99.8^{\circ}$ and once $103^{\circ}$, when fever came on more gradually than usual. The charts show a quasi-normal course, with depressions rather pronounced.

Duration.-This varied from 15 to 50 hours, but the average was near enough the mean of 26 hours to permit this stage being regarded as frequently a well-defined one.

By combining the above two stages the entire incubation-period is obtained; its mean duration was about 90 hours, range from 30 to 126, average rather over the mean, or nearer 4 days than 3 . It varies, in general, with the intensity of ensuing fever; thus, five severe cases had a mean total incubation of 64 hours (range 53 to 84), and four mild cases a mean of 86 (range 66 to 118), these differences being determined chiefly by the length of the second or spirillar stage.

The elements of calculation are submitted in the table appended, from which Experiments 9 and 13 may be eliminated, 17 may be exceptional, and 14 is ill-defined. Most of the data being necessarily approximate, the needed corrections would be an abbreviation of the first with corresponding lengthening of the second stage; estimate of fever was arbitrarily fixed at first observation after rise above mean normal. After correction the second, third, and fourth columns of the table would be still unharmonious, and upon the strength of two experiments more precise than usual (viz. Nos. 2 and 8), a natural variation of the incubation-period, as yet obscurely understood, must be admitted, however unlikely 
this at first sight would appear; upon arrangement of each series a fair average commonly appears, with rarer extremes in either direction, and more cannot now be be advanced. I would only add that these remarks are in accordance with clinical experience.

\section{REMARKS ON THE ANNEXED TABLE.}

\section{(Page 134.)}

The figures in the second to sixth columns are hours; the blank spaces in the second to eighth columns are due to observations cut short or incomplete. The first thirteen experiments were made with the blood of man, the last four with blood of the monkey. The figures in the second column are overstated where the blood examinations were made at long intervals, those in the third column being equally understated. In the fifth column fever is reckoned to begin with rise to and decline until normal upper limits $\left(102 \cdot 3^{\circ}\right)$; comparison with the charts may be made. The fourth column is the sum of the second and third, and the sixth column the sum of the third and fifth.

Incubation-period. - Assuming my data to be free from fallacy, the length of this period is seen to be not only varied in particular instances, but inharmoniously so with regard to current ideas of infection; thus, the three mild attacks after inoculation of human invasion-blood had a mean incubation-period of seventy-five hours, and equally so the two severe and fatal ones of comparative infection at second remove from man (No. 16). This fact seems conclusive of the little significance of incubation-periods as respects the spirillum fever. Nos. 6, 7, 8, and 10 conform in pre-spirillar and apyretic infection, and 11 in the latter only. The longer delay of visible infection in No. 4 (1 Rel.), 12 (2 Rel.), 17 (Incubation), and still more in No. 13, and the two relapses 17 and 12, are not susceptible of collation; if the latter were the result of contagion, the interval in question remains undetermined. No. 9 is wholly exceptional in the other direction.

Fever: Man.-The invasion series Nos. 1, 2, and 3 are concordant, and almost equally brief attacks occurred after inoculation with incubation-blood (Nos. 13 and 17, with relapse). No. 9 had also a brief attack. The sharp rebound of No. 4 is not reckoned above. No. 12 (second relapse) stands alone; the period of spirillar infection (sixth column) was nearly alike in first and second attacks. Monkey.-This series is tolerably concordant, and offers a great contrast to the corresponding invasion-series of man, the mean duration of fever being as 72 to 7 -a prominent fact. 


\begin{tabular}{|c|c|}
\hline 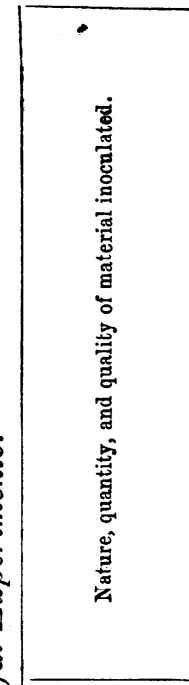 & 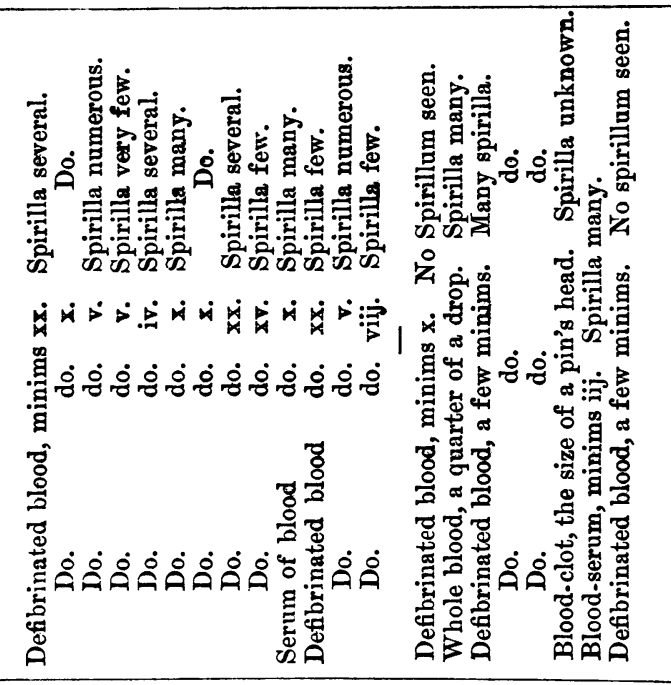 \\
\hline 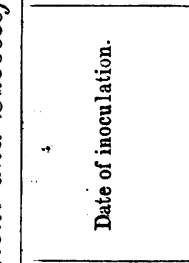 & 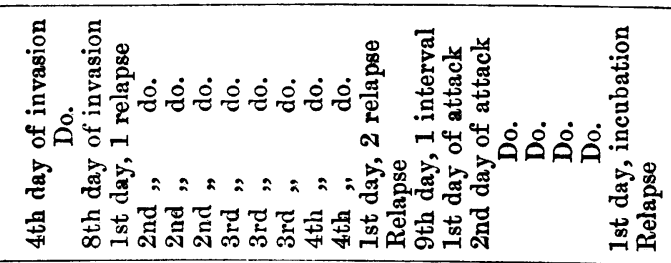 \\
\hline 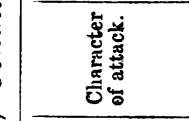 & 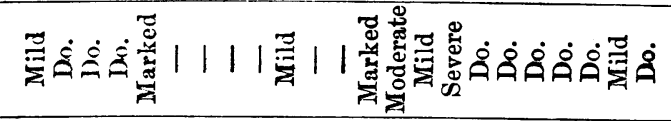 \\
\hline 焉 & 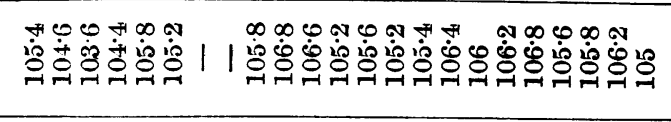 \\
\hline 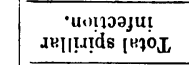 & 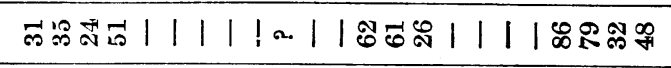 \\
\hline 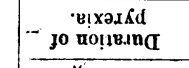 & 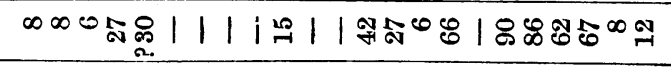 \\
\hline 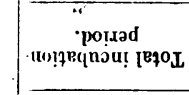 & 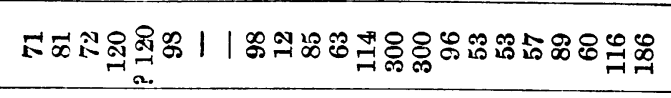 \\
\hline 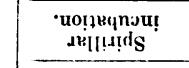 & 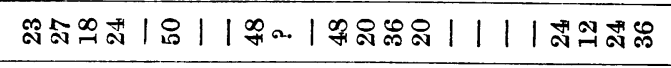 \\
\hline 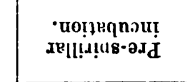 & 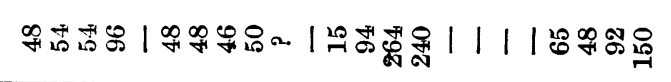 \\
\hline 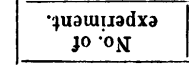 & 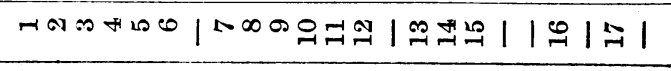 \\
\hline
\end{tabular}




\section{Stage of manifested Fever.}

Beginning.-Fever sets in from $1 \frac{1}{2}$ to 5 days (at most) after inoculation, the mean period being 75 to 80 hours, after excluding an exceptional instance. The hour is commonly near noon or somewhat later, but there is no rule, and it may be at night or early morning; since the majority of inoculations were practised at 5 p.m., no relationship of time here appears. A high temperature is attained on the first day, and generally within a very few hours (3 to 6 ), it may possibly be earlier, and is sometimes later ( 8 to 12 hours); this abrupt beginning of high fever is also a character of the attacks in man. The mean temperature at observed initiation was $104.8^{\circ}$ for a morning rise, and $105^{\circ}$ for an evening rise, both figures being doubtless within the actual, yet sufficient to show how decided is the onset of fever. As the normal temperature of the monkey is higher than that of man by $3^{\circ}$, the absolute initial rise is not so great as in him, yet the upward limit being nearly the same on this first day it is observable that the spirillar pyrexia again displays its special character.

Course.-As to duration, the induced fever is briefer than that of man, never lasting longer than $3 \frac{1}{2}$ days $(86$ hours), in the mean barely 2 days ( 43 hours), and at the shortest only 6 hours. Such very varied duration has no counterpart in the first attack of man, but it accords with the varying length of "relapses" in the human subject; so that the single comparative attack may be said to represent almost all known degrees of fever. Proportionately to its bulk of body the lower animal suffers more than equally, and so does the human infant, hence the hypothesis of a limited materies morbi insita has no application here. One half the attacks might be called mild, the fever lasting not more than 24-30 hours; the 
rest were pronounced, lasting twice or thrice as long, and being sometimes fatal in the rebound. The form and degree of pyrexia may be stated as follows:-Fever is paroxysmal in the briefer attacks, and remittent, or even continuous, in the severer. Five mild instances had a total mean duration of 16 hours, and the form of an isolated paroxysin, declining somewhat more slowly than it rose, and more deeply than its starting-point; the temperature attained was in the mean $104.7^{\circ}$, at maximum $105.8^{\circ}$, and at minimum no more than $103.7^{\circ}$, or not more than $1_{2}{ }^{\circ}$ above the normal level. A rebound was rare, yet prominent once (vide Experiment 4). In two pronounced attacks, lasting $24-48$ hours, the fever was practically continuous, with a depression at early morning hours more or less marked; the second wave was rather less prominent $\left(105 \cdot 2^{\circ}\right)$ than the first $\left(105 \cdot 6^{\circ}\right)$. Five severe attacks lasted from 62 to 86 hours, or in the mean rather over three days; in all, the fever was continuous when viewed at short periods, but when projected from the daily maxima and minima its remittent character was very apparent; high pyrexia was rarely sustained for 24 hours together, and the single instance showing this had a low initial temperature at starting, the animal dying (vide Experiment 15); the remissions generally took place in the early morning, and varied from $1^{\circ}$ to $3^{\circ}$ or $4^{\circ}$. A daily cyclical range was so apparent that it may be confidently looked for in fever lasting two days or longer, and according to the duration will be the number of daily exacerbations and remissions. The mean temperature of these epochs was the following :-First or initial rise $105.5^{\circ}$, first remission $103.1^{\circ}$, second rise $105.8^{\circ}$, decline $103.8^{\circ}$, third and final rise $105.9^{\circ}$, and fall (crisis) $101.7^{\circ}$; there is a visible tendency to gradual augmentation of pyrexia till the acme (maximum temperature $1066^{\circ}$ ) immediately prior to crisis of attack. The mid-febrile remissions amounted in the mean to $2 \cdot 3^{\circ}$ (first) and $2 \cdot 1^{\circ}$ (second), or about one half the final fall.

All these sivere attacks were succeeded by a sharp 
rebound or secondary fever, during which three animals died, and a fourth would probably have succumbed had it been allowed to live as long. Excepting in absolute briefer duration, these comparative phenomena agree with human experiences, and as the contemporary blood-infection is the same, I conclude that the specific pyrexia of man was actually reproduced in the lower animal.

Crisis.-This was always well-defined, yet not so extremely pronounced, or commonly quite so abrupt as in the human prototype. Profuse sweating or incipient collapse were not noticed, and in the milder cases recovery was prompt. The main fall was usually recorded at the 5 a.m. observations; the absolute decline varied from $2.8^{\circ}$ to $6 \cdot 6^{\circ}$, mean about $4^{\circ}$ Fahr., the mimimum temperature noted at this time was hardly below that sometimes recorded in healthy animals $\left(100^{\circ} \mathrm{Fahr}.\right)$, whereas in man it may be $2^{\circ}$ or $3^{\circ}$ lower than normal minimum, and this difference may be referable to the greater constitutional robustness of the ferce.

\section{Relationship of Pyrexia to Incubation-Period.}

No invariable rule was here manifested, only it may be said that whilst for all degrees of fever the earlier nonspirillar stage had a mean duration of near 60 hours, that of apyretic infection had a mean of $26^{\circ}$; divergencies as regards the intensity of ensuing pyrexia were more variable than the conditions of experiment, which was hardly to be anticipated, and I regard the absence of an appreciable regularity of sequence in this respect, an incubation-period of almost identical length preceding, in individual instances, both mild and severe attacks, as indicative of some intrinsic difference of the contagium which is not displayed until fever sets in. The following note bears upon this topic. 
Comparative Severity of Fever in the Monkey.

A mild or severe attack in the human subject does not necessarily entail a corresponding one in the lower animal, but inoculation from a monkey infected directly or at one remove, especially from man, always produced a severe attack ; and hence it appears that the human virus becomes intensified in passing through the monkey. The following tabular statement of results of seventeen inoculations, more or less completely followed out, illustrates this point ; the data are means only, and those of Experiments $9,13,14$, and 17, have been excluded as being least certain or precise.

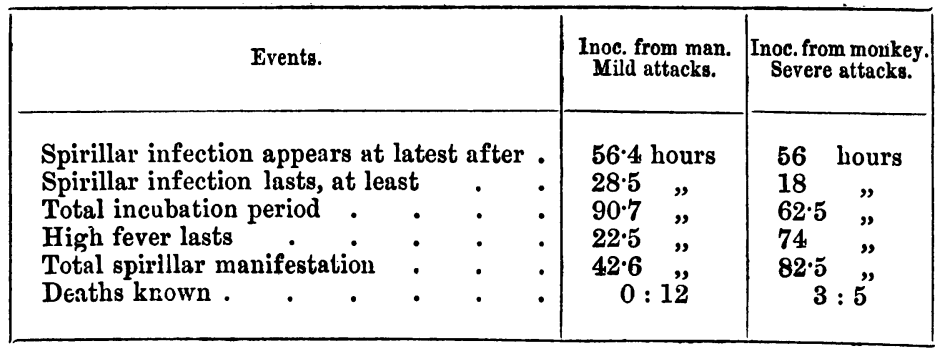

N.B.-Though derived from common data, these figures may seem not to tally with others previously given, but there is no real discordance.

Somewhat parallel with this striking augmentation of infective properties by repetition (if it be nothing more), is that indicated by the experiments made with human blood at successive pyrexial stages of relapsing fever; thus, infection at "invasion" (Nos. 1 to 3), resulted in the mildest form of attack. whilst the "first relapse" supplied more active material (No. 5), and the barely visibly contaminated blood at " second relapse" (No. 12), was also highly effective. Similar results might be looked for in human experience, and if the successive attacks of 
"recurrent typhus" have anything of a cumulative character, or together be comparable with the longer sole attack of ordinary typhus, it might be remembered that the infective powers of the latter fever are greatest at, if not limited to, its terminal stage; nor is the instance a singular one of a contagium slowly gathering in force.

Rebound of Temperature or Secondary Fever.

This is the commoner sort of "relapse" met with, and the phenomenon is a striking one. After mild specific attacks it assumes the form of a smart but brief reaction, and is promptly recovered from; after the severer attacks it is commonly contemporary with local inflammation (see the note on autopsies), and may lead to death. The blood is always free from spirillar contamination here, as in the human subject. Rebounds set in either immediately after the crisis or in the course of a few hours, the milder lasting six hours, the complicated from one and a half to three and a half days, or as long as the prior specific fever. According to their duration, the pyrexia is wholly paroxysmal, or it is sustained and of remittent type, with a tendency to become continued; the onset is prompt and so is the final decline. The temperature attained equals or more commonly exceeds by about $1^{\circ} \mathrm{Fahr}$. that of the specific attack, and the highest recorded in my series, viz. $107 \cdot 2^{\circ}$ occurred at the beginning of secondary fever (see the chart of Experiment No. 15); all the maxima were over $106^{\circ}$; the remissions varied from $1^{\circ}$ to $3.4^{\circ}$. At the decline, body-heat did not descend below normal limits, except in the three fatal cases, when it probably sank till death.

Secondary fever followed all the severer specific attacks seen throughout, and of four instances only one survived after serious illness; its occurrence after milder attacks 
was irregular. Experiment No. 4 is a good example. I need only add that this event furnishes evidence of a similarity, descending even to incidental characters, between the human and comparative attacks of spirillum fever.

Relapses. -The only two known instances have been described in Experiments 13 and 17; they resembled in all features ordinary mild attacks and occurred six and twelve days after the primary event. The extreme range in man was 5 and 12 days in an infinitely larger series than the present, and the average interval was practically invariable, hence my hesitation to insist upon this limited and irregular comparative experience. The absence of a relapse would be no argument against the identity of the spirillum ferer of the monkey with the relapsing fever of man, for in the human subject second attacks are wanting in one fourth of cases, or even oftener, and still the invasion preserves its usual characters. I am not aware that relapses were overlooked in these experiments.

The Tost-mortem appearances in Spirillum-Fever.

Twelve autopsies were made of nine animals killed at various stages, and in three dying of fever. During the incubation-period (three cases) commonly no striking change was noticed, but once deep congestion of the mucous membrane of the stomach, with minute petechiæ, about the middle of the viscus ; liver and spleen congested. In five deaths during fever, vascularity of the stomach about the middle, with small petechiæ, and vascularity of the duodenum and the lower end of the ileum, with congestion of liver and spleen. In one animal bled to death the liver was almost translucent and the spleen small, flabby, and pale, which shows how little the solid parts were altered. Twice, however, there was pulmonary apoplexy; the brain, 
heart, and kidneys were not notably changed. In one death at "full," the viscera showed nothing peculiar. In the three animals dying during secondary fever the liver was congested and once enlarged; the spleen large and congested; kidneys healthy-looking; the mucous membrane of the stomach was once unchanged in aspect, and twice inflamed (being once of a deep maroon colour) about the middle. In two animals dying on the third day of rebound the small intestines were inflamed throughout, beginning abruptly at the pylorus and ending at the ileo-crcal valve, there being hæmorrhagic spots also. In one animal dying a little later, this mucous membrane was very vascular and the intestinal walls were very thin; the large intestine was comparatively unchanged, the rectum only being rather vascular. Petechiæ were seen twice on the lungs and on the heart in these three cases; the brain was pallid only. These data form part of the evidence showing the identity of the "fever" in the man and animal.

After death from poisoning by saliva (two cases) inflammation of the stomach and extravasation into the arachnoidal sac were once noted.

Portions of the above viscera are preserved, and may show minute textural changes (possibly in the bloodvessels), elucidating the essential seat of morbid lesion better than coarser appearances; at present, I have detected no peculiar changes except within the blood.

Microscopic Observations on the Blood.

From a very large number of data I am able to state that there obtains the closest correspondence in aspect between the blood of the monkey and of man at all stages of the spirillum fever, including that of apyretic infection; nor has any discordance come to light. Respecting the less peculiar constituents, various forms of protoplasm deserve 
mention from their possible connection with development of the spirillum ; but as such "clumps" and "filaments" may be independent, attention will here be limited to the parasite alone.

I regard it as manifest that this organism is identical in both species, and its comparative prevalence in time is also alike. Thus, on first detection in the early nonfebrile stage its numbers are sparse (e.g. one or two in field of view), and being only rather commoner in the monkey; it then has a fully-formed aspect and would seem to be introduced into the blood $a b$ extra; no change occurs until the onset of fever. The spirillum may, indeed, somewhat diminish in numbers, and even temporarily disappear at this time, but with, or even before the rise of temperature, it rapidly augments (e.g. six to twelve in the field), and hence sometimes I have been able to predict the advent of fever. During the course of pronounced pyrexia the parasite positively swarms in the monkey's blood (appearing almost as numerous as the red discs); it is little less abundant with remissions of temperature, but it promptly and wholly disappears at a period, sometimes computable by minutes, immediately prior to initiation of the critical fall of temperature, vanishing at the perturbatio critica when this phenomenon occurs. The intermittent appearance of the spirillum during high fever, and its continuance at a complete intermission of temperature or during the fall by "lysis," are exceptional phenomena hitherto seen only in man; and the inevitable inference from my comparative studies is wholly in favour of there being the closest relationship between abounding spirillar infection and pronounced pyrexia. The evidence of interrupted parasitic growth, or accession by "crops" or "broods," has not been so manifest here as in the longer attacks of man.

The great variety of form assumed by the more active spirillum in the freshest possible blood does not seem to be remembered, and the ordinary delineation of a straight spiral flament pertains rather to a more quiescent state 
supervening some time after withdrawal from the body; in quickly abstracted blood I found the organism in inces. sant movement, presenting the shape of curved and twisted filaments, and of loops, knots, and rings, such as wore never assumed by the spirilla and spirochæte of tankwater, which I also submitted to scrutiny for comparison. These last preserve their rigid spiral contour in all their movements, and do not unfold, as it were. On the other hand, I have seen the Bacillus (subtilis ?) of similar water part with its straight or wary outline, and take on a twisted, spiral form, which evidenced nearly as much flexibility and extensibility or contraction as is shown by the blood parasite, and my impression is that the latter organism might be defined anew.

The spirillum of the saliva has been alluded to already in Experiment 42.

For thorough scrutiny of the blood it is necessary to employ the Albrecht process, with or without subsequent staining; and simple dyeing of the blood elements with anilin violet will also be found very useful. By either of these means a multiplication of the spirillum by mid-fission may be seen in most specimens of infected blood, and oftenest, I think, when the parasite is increasing. Often, too, the presence of immature and possibly growing organisms is thus made apparent, the primitive granules and short curved filaments having a diameter somewhat larger than that of the perfect parasite. A dotted or even partially beaded condition of the latter may sometimes be noted, which seems indicative of a mode of growth. The same processes applied to the venous bloods issuing from the spleen, liver, lungs, kidney, \&c., during the height of fever, reveal a striking aspect of the plasmic contents, which I can only interpret as significant of active spirillar development; the splenic venous blood is especially rich in endothelial and white-cell forms containing the germs (?) and incipient spiral filaments; this subject is still under inquiry. I should add that there are some fallacious appearances pertaining to blood protoplasm, and (in Bom. 
bay, at least) to minute incidental fungus growth which require discrimination.

As to the mode of disappearance of the spirillum at the close of fever, this organism has been found in preserved specimens of blood to quickly melt away leaving no imme. diate trace behind; sometimes the amœboid masses common in the plasma seemed to involve and remove the quiescent filaments. That the destruction of the parasite, now or previously, is an active process was shown by the fact that in specimens of infected blood kept for several hours the spirillum had been preserved in activity outside the body, whilst it had altogether disappeared from blood drawn afresh within the same period, having evidently been destroyed by some natural process not operating outside the living frame. It was not clear that this process pertained only to a high temperature.

The activity and life of the spirillum are independent of the life of its host ; in the living subject this organism is to be seen only in active movement, and thus its detection is rendered comparatively easy. Its movements may persist for many hours after decease of man or monkey (e.g. 24 or 36, ), and their cessation is not a sign of its own death, for they cease before the filament begins to grow under the influence of artificial culture.

Reserving other details, I will only add here that the parasite of spirillum fever may be readily induced to multiply and grow into a delicate mycelioid network, which in favorable conditions is very luxuriant and striking. Sometimes spores seemed to arise from this fructification, but I was never able to see the production of a second generation of free spiral organisms.

On the connection between the results of Insculation and of Artificial Cultivation of the Blood.

There exists a certain accord between the experimental series above described and another carried out about the 
same time and with the same or similar material, in which attempts were made to induce growth or reproduction of the spirillum outside the body, and the results are worthy of brief mention here, even if it be not admitted that the blood parasite is the "cause" of fever.

Thus, in eight essays at culture I found the spirillum is never developed in blood not containing it at the beginning of experiment, and this is in accordance with the negative results of inoculation practised with similar material. It would, therefore, appear that the germs of the parasite are not habitually present in the blood.

Again, in eighteen cultivation experiments made with visibly infected blood, some (eight) failed whilst others (ten) succeeded, just as is the case with inoculation experiments which are only partially successful. The failures were two of incubation-blood, one of invasion-blood at "acme," one of invasion-blood at "fall," both specimens taken from the same patient as in Experiment 18, which was also unsuccessful at the last of these dates; three failures were of blood at first day of "relapse," which is in accordance with Experiments 21 and 22, and the blood employed was the same as in No. 22 ; one was of relapseblood at fall, which also accords; and, in short, these negative data agree throughout, nor are there discrepancies. It therefore appears that growth of the parasite within the blood is, at least, not usual at certain stages of the fever. The successes were six, viz. four of invasionblood of man from second to seventh day inclusive, and two of monkey's blood at first and second day of high fever, both series agreeing with positive inoculations. Since success in culture implies the growth and increase of spirilla in the medium employed, its relationship with successful inoculation becomes obvious; and the inference favours the view of the blood parasite being essentially connected with the production of pyrexia. 


\section{Conclusions.}

1. "Relapsing fever" is readily transmissible from man to a quadrumanous animal, and from one of these animals to another, by inoculation of the blood, and it then commonly assumes the form of a single febrile event of highly varied intensity, type, and duration. So far as evidence goes the "relapse" is much more incidental in the monkey than in man. The conditions of infection are multiple.

2. In the comparative attack the incubation-period is characterised towards its close by a non-febrile spirillar infection of the blood, and the same fact has been verified for the first apyretic interval of man. It therefore appears that all incubation-periods are divisible into two stages of a prior non-specific and a later specific character respectively. The duration of these periods, or even of their stages, bears no fixed proportion to the intensity of ensuing febrile phenomena.

3. The relationship of spirillar blood-infection to pyrexia cannot hence be regarded as an immediate one, yet, in the monkey, fever was an invariable sequel to infection, beginning and being conterminous with, and in degree generally corresponding to, augmentation of the parasite in the blood. In milder degrees of infection, however, it is conceivable that the terminal development of pyrexia may be wanting, and hence the spirillum disease would become essentially definable as a mycosis sanguinis prope cum febre.

4. Nothing definite has been elicited respecting contemporary pyrogenetic agencies of a specific character, other than the spirilla. Such there may be, yet the tendency of my comparative observations was towards the inference that exceptions to the relationship indicated are explicable upon idiosyncrasy of subject or other complication, the non-specific causes of "ferer" being numerous. 


\section{APPENDIX.}

Successful Inoculation with Blood of Monkey at INCUBATION-PERIOD.

M. No. 1, new animal, had five minims of defibrinated blood taken from a woman, æt. 35, at last day (a.m.) of a characteristic invasion-attack of relapsing fever (temp. $104.8^{\circ} \mathrm{F}$.), injected hypodermically in the thigh ; material employed contained a great number of active and quiescent spirilla; blood and temperature of the monkey normal.

On the second day at 4 a.m., or 16 hours after injection, the parasite was found in the animal's blood (temp. $99 \cdot 2^{\circ}$ ), and for 48 hours longer there was no fever; then a brief intermittent attack ensued, marked, as usual, with increased visible contamination of the blood, and ending with abrupt fall and concurrent disappearance of the spirillum (vide Chart, Diagram VIII).

M. No. 2, a female, freshly brought from the bazaar, had hypodermically injected in the thigh ten minims of entire blood drawn from the ear of No. 1 about midperiod of non-febrile, specific incubation-stage (vide Chart, Diagram VIII). At this time very few spirilla were to be seen in the blood employed for inoculation, even on testing with the acetic acid process. For two days (possibly less) there was no change in No. 2, but on the fourth day, or 64 hours after experiment (temp. $101 \cdot 2^{\circ}$ ), a single organism was detected in its blood, and thenceforward others for 48 hours longer, still without fever, when a brief febrile paroxysm occurred, which terminated the attack.

There were no peculiarities in the state of the blood, temperature, or general symptoms of this case, which seems to show conclusively the possibility of infection during the incubation-stage of fever, due precautions having been taken to avoid error from incidental contagion. 
148 EXPERIMENTAL PATHOLOGY OF SPIRILLUM FEVER.

Previously negative results are therefore qualified by this positive issue, and the significance of the spirillar state is, perhaps, increased thereby.

Вомвцт ; December, 1879. 